\title{
Eigenvalues for double phase variational integrals
}

\author{
Francesca Colasuonno $^{2} \cdot$ Marco Squassina $^{1}$
}

Received: 7 July 2015 / Accepted: 14 October 2015 / Published online: 29 October 2015

(C) Fondazione Annali di Matematica Pura ed Applicata and Springer-Verlag Berlin Heidelberg 2015

\begin{abstract}
We study an eigenvalue problem in the framework of double phase variational integrals, and we introduce a sequence of nonlinear eigenvalues by a minimax procedure. We establish a continuity result for the nonlinear eigenvalues with respect to the variations of the phases. Furthermore, we investigate the growth rate of this sequence and get a Weyl-type law consistent with the classical law for the $p$-Laplacian operator when the two phases agree.
\end{abstract}

Keywords Quasilinear problems · Double phase problems · Nonstandard growth conditions · Musielak-Orlicz spaces $\cdot \Gamma$-convergence $\cdot$ Stability of eigenvalues .

Weyl-type laws

Mathematics Subject Classification $35 \mathrm{~J} 92 \cdot 35 \mathrm{P} 30 \cdot 47 \mathrm{~A} 75$

\section{Introduction}

\subsection{Overview}

While the theory of linear eigenvalue problems is a well-established topic of functional analysis [16], in the last few decades many contributions were devoted to the study of nonlinear eigenvalue problems. As pointed out by P. Lindqvist in his monograph [44], the work [37] by E.H. Lieb was probably one of the first containing an interesting result about the minimum of a nonlinear Rayleigh quotient in several variables. Subsequently, and especially in the first

Marco Squassina

marco.squassina@univr.it

Francesca Colasuonno

f.colasuonno@iac.cnr.it

1 Dipartimento di Informatica, Università degli Studi di Verona, Cá Vignal 2, Strada Le Grazie 15, 37134 Verona, Italy

2 Istituto per le Applicazioni del Calcolo "M. Picone", Consiglio Nazionale delle Ricerche, Via dei Taurini 19, 00185 Rome, Italy 
years of the nineties, various papers were written by P. Lindqvist on the subject, we recall here [28,38,40-42] and the comprehensive overview contained in his monograph [44].

\subsection{1 p-Laplacian}

More precisely, if $\Omega \subset \mathbb{R}^{n}$ is a smooth bounded domain and $p>1$, for the quasilinear eigenvalue problem

$$
-\operatorname{div}\left(|\nabla u|^{p-2} \nabla u\right)=\lambda|u|^{p-2} u, \quad u \in W_{0}^{1, p}(\Omega),
$$

existence, regularity, qualitative properties, and stability of eigenpairs $(u, \lambda)$ with respect to $p$ were investigated. The physical motivations that lead to the study of the eigenvalue problem (1.1) are mainly within the context of non-Newtonian fluids, dilatant for $p>2$ and pseudoplastic for $1<p<2$, nonlinear elasticity and glaciology. Of particular relevance is the investigation of the properties of the first eigenpair $\left(u_{p}^{1}, \lambda_{p}^{1}\right)$, which corresponds to a solution to the nonlinear minimization problem

$$
\lambda_{p}^{1}(\Omega):=\inf _{u \in \mathcal{M}_{p}}\|\nabla u\|_{p}^{p}, \quad \mathcal{M}_{p}:=\left\{u \in W_{0}^{1, p}(\Omega):\|u\|_{p}=1\right\} .
$$

In the one-dimensional case, $\left(u_{p}^{1}, \lambda_{p}^{1}\right)$ is explicitly determined by solving the corresponding ODE boundary value problem. If $\Omega=(a, b)$, then

$$
\lambda_{p}=\left(\pi_{p} /(b-a)\right)^{p-1}, \quad \pi_{p}:=2(p-1)^{1 / p} \int_{0}^{1}\left(1-s^{p}\right)^{-1 / p} \mathrm{~d} s,
$$

and

$$
u_{p}^{1}(x)=(p-1)^{-1 / p} \sin _{p}\left(\pi_{p}(x-a) /(b-a)\right),
$$

where $\sin _{p}$ is a $2 \pi_{p}$-periodic function that generalizes the classical sine function [43]. Of course an analogous analysis is not possible in the higher-dimensional case. The existence of a sequence of higher eigenvalues $\left(u_{p}^{m}, \lambda_{p}^{m}\right)$ can be obtained as a solution to

$$
\lambda_{p}^{m}(\Omega):=\inf _{K \in \mathcal{W}_{p}^{m}} \max _{u \in K}\|\nabla u\|_{p}^{p}
$$

where

$$
\mathcal{W}_{p}^{m}:=\left\{K \subset \mathcal{M}_{p}: K \text { symmetric }(K=-K) \text { and compact, } \gamma(K) \geq m\right\},
$$

and $\gamma(K)$ denotes the Krasnosel'skiu genus of $K$. We recall that for every nonempty and symmetric subset $A$ of a Banach space $X$, its Krasnosel'skiu genus is defined by

$$
\gamma(A):=\inf \left\{k \in \mathbb{N}: \exists \text { a continuous odd map } f: A \rightarrow \mathbb{S}^{k-1}\right\},
$$

where $\mathbb{S}^{k-1}$ is the unit sphere in $\mathbb{R}^{k}$, and with the convention that $\gamma(A):=+\infty$, if no such an integer $k$ exists. Actually one can define, in a similar fashion, a sequence of higher variational eigenvalues by replacing the Krasnosel'skiu genus with any other topological index $i$ which satisfies the properties listed at the end of Sect. 4. It is unknown whether these topological constructions exhaust the spectrum or not, which is the case for linear eigenvalue problems. This is, in fact, one of the main open problems in the field since the appearance of these results. The sequence of eigenvalues $\left(\lambda_{p}^{m}\right)$ depend continuously, in smooth domains, on the value of $p$ (cf. $[12,21,22,40,42,50])$ and, fixed the value of $p$, they grow in $m$ according to a suitable Weyl-type law, $\lambda_{p}^{m} \approx C m^{p / n}$ for $m$ large, consistently with the celebrated Weyl 
law for the linear case $p=2$, see e.g., [29,31]. For a complete investigation of the Stekloff spectrum for the pseudo $p$-Laplacian operator $\sum_{i} D_{i}\left(\left|D_{i} u\right|^{p-2} D_{i} u\right)$, we refer the reader to [10].

\subsubsection{Fractional p-Laplacian}

We wish to point out that, very recently, a nonlocal version of the $p$-Laplacian, the fractional $p$-Laplacian $\left(-\Delta_{p}\right)^{s}$,

$$
\left(-\Delta_{p}\right)^{s} u(x):=2 \lim _{\varepsilon \searrow 0} \int_{\mathbb{R}^{n} \backslash B_{\varepsilon}(x)} \frac{|u(x)-u(y)|^{p-2}(u(x)-u(y))}{|x-y|^{n+s p}} \mathrm{~d} y, \quad x \in \mathbb{R}^{n} .
$$

was introduced in [38], where properties of the first eigenvalue are investigated. Subsequently, Weyl-type laws were studied in [33] (an optimal result, consistent with the local case, is not available yet, due to the strong nonlocal effects in the analysis) and a complete analysis about the stability of variational eigenvalues was obtained in [8], with particular reference to the singular limit $s \nearrow 1$ toward the eigenvalues of the fractional Laplacian $(-\Delta)^{s}=$ $\mathcal{F}^{-1} \circ \mathscr{M}_{s} \circ \mathcal{F}$, where $\mathcal{F}$ is the Fourier transform operator and $\mathscr{M}_{s}$ is the multiplication by $|\xi|^{2 s}$. A rather complete analysis about the properties of the second eigenvalue was carried on in [9].

\subsection{3 $p(x)$-Laplacian}

Motivated by nonlinear elasticity theory and electrorheological fluids, problems involving variable exponents $p(x)$ were also investigated, especially in regularity theory (see e.g., [1,2, 23 ] and the references therein). Quite recently in [28], nonlinear eigenvalues were investigated in this framework. If $p: \bar{\Omega} \rightarrow \mathbb{R}^{+}$is a log-Hölder continuous function and

$$
1<p_{-}:=\inf _{\Omega} p \leq p(x) \leq \sup _{\Omega} p=: p_{+}<n \quad \text { for all } x \in \Omega,
$$

the $m$-th (variational) eigenvalue $\lambda_{p(x)}^{m}$ can be obtained as

$$
\lambda_{p(x)}^{m}:=\inf _{K \in \mathcal{W}_{p(x)}^{m}} \sup _{u \in K}\|\nabla u\|_{p(x)}
$$

where $\|\cdot\|_{p(x)}$ is the Luxemburg norm defined by

$$
\|u\|_{p(x)}:=\inf \left\{\gamma>0: \int_{\Omega}\left|\frac{u(x)}{\gamma}\right|^{p(x)} \mathrm{d} x \leq 1\right\} .
$$

and $\mathcal{W}_{p(x)}^{m}$ is the set of symmetric, compact subsets of

$$
\mathcal{M}_{p(x)}:=\left\{u \in W_{0}^{1, p(x)}(\Omega):\|u\|_{p(x)}=1\right\}
$$

such that $i(K) \geq m$, where $i$ denotes the genus or any other topological index satisfying properties $\left(i_{1}\right)-\left(i_{4}\right)$ listed in Sect. 4 . In [28] existence and properties of the first eigenfunction were studied. The stability with respect to uniform perturbations of $p(x)$ was recently investigated in [13] (see also [7]). Finally, the growth rate of the sequence in (1.5) was investigated in [52], getting a natural replacement for the constant case. 


\subsubsection{Double phases}

Given two constant exponents $q>p>1$, one can think about the case of a variable exponent $p(x)$ being a smooth approximation of a discontinuous exponent $\bar{p}: \Omega \rightarrow(1, \infty)$ with $\bar{p}(x)=p$ if $x \in \Omega_{1}$ and $\bar{p}(x)=q$ if $x \in \Omega_{2}$, where $\Omega=\Omega_{1} \cup \Omega_{2}$. In some sense, this situation can be interpreted as a double phase behavior in two disjoint sub-domains of $\Omega$. A different kind of double phase situation occurs for the energy functional

$$
u \mapsto \int_{\Omega} \mathcal{H}(x,|\nabla u(x)|) \mathrm{d} x, \quad \mathcal{H}(x, t):=t^{p}+a(x) t^{q}, \quad q>p>1, a(\cdot) \geq 0,
$$

where the integrand switches two different elliptic behaviors. This defined in (1.6) belongs to a family of functionals that Zhikov introduced to provide models of strongly anisotropic materials, see $[60,62,63]$ or [61] and the references therein. Also, (1.6) settle in the context of the so-called functionals with nonstandard growth conditions, according to a well-established terminology which was introduced by Marcellini [45,46], see also [17-19,47]. In [61], functionals (1.6) are used in the context of homogenization and elasticity and the function $a$ drives the geometry of a composite of two different materials with hardening powers $p$ and $q$.

Significant progresses were recently achieved in the framework of regularity theory for minimizers of this class of integrands of the Calculus of Variations, see e.g., [3-5, 14, 15].

\subsection{Main results}

The main goal of this paper is to introduce a suitable notion of eigenpair associated with the energy functional (1.6) consistently with the case $p=q$ and $a \equiv 1$, to prove the existence of an unbounded sequence of eigenvalues and, furthermore, get continuity of each of these eigenvalues with respect to $(p, q)$ and a Weyl-type law consistent with the classical (single phase) case. Let $W_{0}^{1, \mathcal{H}}(\Omega)$ be the Musielak-Orlicz space introduced in Sect. 2 and

$$
\mathcal{M}_{\mathcal{H}}:=\left\{u \in W_{0}^{1, \mathcal{H}}(\Omega):\|u\|_{\mathcal{H}}=1\right\},
$$

where $\Omega$ is a bounded domain of $\mathbb{R}^{n}$. In the sequel, we shall denote this set simply by $\mathcal{M}$. In the next theorems, we assume that $1<p<q<n$ and that the following condition holds

$$
\frac{q}{p}<1+\frac{1}{n}, \quad \partial \Omega \quad \text { and } \quad a: \bar{\Omega} \rightarrow[0, \infty) \text { are Lipschitz continuous. }
$$

The following are the main results of the paper.

Theorem 1.1 (The first eigenpair) The first eigenvalue

$$
\lambda_{\mathcal{H}}^{1}:=\inf _{u \in \mathcal{M}}\|\nabla u\|_{\mathcal{H}}
$$

is positive and there exists a positive minimizer $u_{\mathcal{H}}^{1} \in \mathcal{M} \cap L^{\infty}(\Omega)$ which solves

$$
-\operatorname{div}\left(p\left|\frac{\nabla u}{\lambda}\right|^{p-2} \frac{\nabla u}{\lambda}+q a(x)\left|\frac{\nabla u}{\lambda}\right|^{q-2} \frac{\nabla u}{\lambda}\right)=\lambda S(u)\left(p|u|^{p-2} u+q a(x)|u|^{q-2} u\right),
$$


with $\lambda=\lambda_{\mathcal{H}}^{1}$, where

$$
S(u):=\frac{\int_{\Omega}\left(p\left|\frac{\nabla u}{\lambda}\right|^{p}+q a(x)\left|\frac{\nabla u}{\lambda}\right|^{q}\right) d x}{\int_{\Omega}\left(p|u|^{p}+q a(x)|u|^{q}\right) d x} .
$$

The first eigenvalue $\lambda_{\mathcal{H}}^{1}$ is stable under monotonic perturbations of $\Omega$. If $a \equiv 1$, furthermore, balls uniquely minimize the first eigenvalue among sets with a given n-dimensional Lebesgue measure. Finally, if $a \equiv 1$ and, given a polarizer $H$ with $0 \in H$ (resp. $0 \in \partial H$ ), the domain $\Omega$ coincides with its polarization $\Omega^{H}$ (resp. its reflection $\Omega_{H}$ ), then there exists a first nonnegative eigenfunction having the symmetry $u=u^{H}$.

The previous theorem is a consequence of a Poincaré-type inequality, which has been proved in the general framework of Musielak-Orlicz spaces in [26,32].

It is important to stress that, due to the presence of the term $S(u)$, Eq. (1.8) turns out to be nonlocal. A similar nonlocal character arises in the case of the $p(x)$-Laplacian.

It would be interesting to investigate the simplicity of the first eigenvalue as well as understanding if an arbitrary eigenfunction of fixed sign is automatically a first eigenfunction. These issues are known to hold in the cases of the $p$-Laplacian and the fractional $p$-Laplacian, but, to the authors' knowledge, no result seems to be available in inhomogeneous settings like the $p(x)$-Laplacian or the double phase operators.

In what follows, $i$ denotes the genus or any topological index satisfying $\left(i_{1}\right)-\left(i_{4}\right)$ in Sect. 4.

Theorem 1.2 (Nonlinear spectrum). If $\mathcal{W}_{\mathcal{H}}^{m}$ is the set of symmetric compacts $K$ of $\mathcal{M}$ with index $i(K) \geq m$, then the sequence

$$
\lambda_{\mathcal{H}}^{m}:=\inf _{K \in \mathcal{W}_{\mathcal{H}}^{m}} \sup _{u \in K}\|\nabla u\|_{\mathcal{H}},
$$

is nondecreasing, divergent, and for every $m \geq 1$ there exists $u_{m} \in \mathcal{M}$ solving Eq. (1.8), with $\lambda=\lambda_{\mathcal{H}}^{m}$.

The result provides the construction of a variational spectrum for the double phase integrands which is consistent with the single phase case $p=q$, see e.g., [21,22]. The proof relies on the properties of the topological index $i$ and is based on the fact that the even functional $\left.K\right|_{\mathcal{M}}: u \in \mathcal{M} \mapsto\|\nabla u\|_{\mathcal{H}}$ satisfies the (PS) condition. As for the $p$-Laplacian, it is not known whether the variational spectrum (i.e., the sequence of variational eigenvalues $\left(\lambda_{\mathcal{H}}^{m}\right)$ ) exhausts the whole spectrum (i.e., the set of all eigenvalues of (1.8), see Definition 3.3) or not. In Theorem 3.12, we prove that the spectrum is a closed set. While the following two results concern only the variational spectrum.

Theorem 1.3 (Stability) Let $\left(p_{h}, q_{h}\right) \searrow(p, q)$ as $h \rightarrow \infty$. Then

$$
\lim _{h \rightarrow \infty} \lambda_{\mathcal{H}_{h}}^{m}=\lambda_{\mathcal{H}}^{m} \quad \text { for every } m \geq 1,
$$

where $\mathcal{H}_{h}(x, t):=t^{p_{h}}+a(x) t^{q_{h}}$ and $\mathcal{H}(x, t):=t^{p}+a(x) t^{q}$.

The result implies, in particular, that each element of the sequence of nonlinear eigenvalues $\left(\lambda_{\mathcal{H}_{h}}^{m}\right)$ for the double phase case converges to the corresponding nonlinear eigenvalue for the $p_{0}$-Laplacian operator, whenever $\left(p_{h}, q_{h}\right) \searrow\left(p_{0}, p_{0}\right)$, as $h \rightarrow \infty$. The proof of Theorem 1.3 uses some recent results of [21] and involves the $\Gamma$-convergence of a class of even functionals defined in $L^{1}(\Omega)$. 
In what follows, for any $E \subset \mathbb{N}$, we shall denote by $\sharp E$ the number of elements of $E$. Furthermore, $A \subset \mathbb{R}^{n}$ is called quasiconvex if there exists a constant $C>0$ such that for all $x, y \in A$ there is an arc joining $x$ to $y$ in $A$ having length at most $C|x-y|$.

Theorem 1.4 (Weyl law) Let $\Omega$ be quasiconvex and let us set

$$
w:=1+\|a\|_{\infty}+|\Omega|, \quad \sigma:=n\left(\frac{1}{p}-\frac{1}{q}\right) .
$$

Let $\lambda_{\mathcal{H}}^{m}$ be defined either through the genus $\gamma$ or through the $\mathbb{Z}_{2}$-cohomological index $g$. Then, there exist $C_{1}, C_{2}>0$, depending only on $n, p, q$, such that

$$
C_{1}|\Omega|(\lambda / w)^{n /(1+\sigma)} \leq \sharp\left\{m \in \mathbb{N}: \lambda_{\mathcal{H}}^{m}<\lambda\right\} \leq C_{2}|\Omega|(w \lambda)^{n /(1-\sigma)},
$$

for $\lambda>0$ large. In particular there exist $D_{1}, D_{2}>0$ depending on $n, p, q$, a and $|\Omega|$ with

$$
D_{1} m^{(1-\sigma) / n} \leq \lambda_{\mathcal{H}}^{m} \leq D_{2} m^{(1+\sigma) / n}
$$

for every $m \geq 1$ large enough.

The result provides a consistent extension of the Weyl-type law for the $p$-Laplacian. We point out that, in the limiting case when $p=q$ and $a \equiv 1$, the Euler-Lagrange Eq. (1.8) [see also the formulation (3.3)] reduces to the usual quasilinear problem (1.1) involving $-\Delta_{p}$, but the eigenvalues $\lambda_{\mathcal{H}}^{m}$ and $\lambda_{p}^{m}$, in light of their definition, do satisfy

$$
\lambda_{\mathcal{H}}^{m}=\left(\lambda_{p}^{m}\right)^{\frac{1}{p}} \quad \text { for all } m \in \mathbb{N}
$$

Hence, the estimate for the growth of $\left(\lambda_{\mathcal{H}}^{m}\right)$ of Theorem 1.4 for the case $p=q$ (formally corresponding to $\sigma=0$ )

$$
D_{1} m^{1 / n} \leq \lambda_{\mathcal{H}}^{m} \leq D_{2} m^{1 / n}
$$

should be compared with the estimate $D_{1} m^{p / n} \leq \lambda_{p}^{m} \leq D_{2} m^{p / n}$ and, thus, it is consistent with the results obtained in $[29,31]$. For the linear case $q=p=2$ and $a=1$, we mention the pioneering contribution by Weyl [58], from which these types of estimates inherit the name. The proof of Theorem 1.4 relies on the properties and on the relations among three different topological indices (i.e., the genus, the cogenus, and the cohomological index) and is an adaptation to the double phase setting of an idea developed in [52] for the $p(x)$-Laplacian operator.

\subsection{Plan of the paper}

In Sect. 2, we give some basic definitions and useful results on Musielak-Orlicz spaces and in particular on the spaces generated by the $N$-function $\mathcal{H}$ as in (1.6). In Sect. 3, we derive the Euler-Lagrange equation corresponding to the minimization of the Rayleigh ratio $\|\nabla u\|_{\mathcal{H}} /\|u\|_{\mathcal{H}}$ and we prove Theorem 1.1 concerning the first eigenpair $\left(\lambda_{\mathcal{H}}^{1}, u_{\mathcal{H}}^{1}\right)$ and some useful properties of the spectrum, such as its closedness and the behavior of the first eigenvalue for large exponents $p$ and $q$. Section 4 contains the definition of the variational eigenvalues of (1.8) and the proof of Theorem 1.2, while Sect. 5 is devoted to the proof, via $\Gamma$-convergence, of the stability of the nonlinear spectrum (Theorem 1.3), i.e., the continuity of the eigenvalues with respect to the variation of the phases $p$ and $q$ from the right. Finally, in Sect. 6 we study the asymptotic growth of the variational eigenvalues and prove Theorem 1.4. 


\section{Preliminary results}

\subsection{Musielak-Orlicz spaces}

We recall here some notions on Musielak-Orlicz spaces, see for reference [48], Section 2 of [23], and also Section 1 of [27]. Let $\Omega \subset \mathbb{R}^{n}$ be a bounded domain.

Definition 2.1 A continuous, convex function $\varphi:[0, \infty) \rightarrow[0, \infty)$ is called $\Phi$-function if $\varphi(0)=0$ and $\varphi(t)>0$ for all $t>0$.

A function $\varphi: \Omega \times[0, \infty) \rightarrow[0, \infty)$ is said to be a generalized $\Phi$-function, denoted by $\varphi \in \Phi(\Omega)$, if $\varphi(\cdot, t)$ is measurable for all $t \geq 0$ and $\varphi(x, \cdot)$ is a $\Phi$-function for a.a. $x \in \Omega$.

$\varphi \in \Phi(\Omega)$ is locally integrable if $\varphi(\cdot, t) \in L^{1}(\Omega)$ for all $t>0$.

$\varphi \in \Phi(\Omega)$ satisfies the $\left(\Delta_{2}\right)$-condition if there exist a positive constant $C$ and a nonnegative function $h \in L^{1}(\Omega)$ such that

$$
\varphi(x, 2 t) \leq C \varphi(x, t)+h(x) \text { for a.a. } x \in \Omega \text { and all } t \in[0, \infty) .
$$

Let $\varphi, \psi \in \Phi(\Omega)$. The function $\varphi$ is weaker than $\psi$, denoted by $\varphi \preceq \psi$, if there exist two positive constants $C_{1}, C_{2}$ and a nonnegative function $h \in L^{1}(\Omega)$ such that

$$
\varphi(x, t) \leq C_{1} \psi\left(x, C_{2} t\right)+h(x) \text { for a.a. } x \in \Omega \text { and all } t \in[0, \infty) .
$$

Given $\varphi \in \Phi(\Omega)$, the Musielak-Orlicz space $L^{\varphi}(\Omega)$ is given by

$$
L^{\varphi}(\Omega):=\left\{u: \Omega \rightarrow \mathbb{R} \text { measurable }: \exists \gamma>0 \text { s.t. } \varrho_{\varphi}(\gamma u)<\infty\right\},
$$

where

$$
\varrho_{\varphi}(u):=\int_{\Omega} \varphi(x,|u|) \mathrm{d} x
$$

is the modular, while

$$
\|u\|_{\varphi}:=\inf \left\{\gamma>0: \varrho_{\varphi}(u / \gamma) \leq 1\right\}
$$

is the norm defined on $L^{\varphi}(\Omega)$.

Proposition 2.2 (cf. [48, Theorem 7.7]) Let $\varphi \in \Phi(\Omega)$, then $\left(L^{\varphi},\|\cdot\|_{\varphi}\right)$ is a Banach space.

Proposition 2.3 (cf. [48, Theorem 8.5]) Let $\varphi, \psi \in \Phi(\Omega)$, with $\varphi \preceq \psi$. Then

$$
L^{\psi}(\Omega) \hookrightarrow L^{\varphi}(\Omega) .
$$

Proposition 2.4 (unit ball property) Let $\varphi \in \Phi(\Omega)$, then the following properties hold.

(i) If $\varphi$ satisfies $\left(\Delta_{2}\right)$, then $L^{\varphi}(\Omega)=\left\{u: \Omega \rightarrow \mathbb{R}\right.$ measurable $\left.: \varrho_{\varphi}(u)<\infty\right\}$, (cf. Theorem 8.13 of [48]).

(ii) If $u \in L^{\varphi}(\Omega)$, then $\varrho_{\varphi}(u)<1$ (resp. $\left.=1 ;>1\right) \Leftrightarrow\|u\|_{\varphi}<1$ (resp. $=1$; > 1), (cf. Lemma 2.1.14 of [23]).

Remark 2.5 As a consequence of the homogeneity of the norm and of the unit ball property (Proposition 2.4-(ii)), we have the following implications:

$$
\|u\|_{\varphi_{1}}=\|v\|_{\varphi_{2}} \Rightarrow\left\|\frac{u}{\|v\|_{\varphi_{2}}}\right\|_{\varphi_{1}}=1 \Rightarrow \varrho_{\varphi_{1}}\left(\frac{u}{\|v\|_{\varphi_{2}}}\right)=1=\varrho_{\varphi_{2}}\left(\frac{v}{\|v\|_{\varphi_{2}}}\right) .
$$


Definition 2.6 For $\varphi \in \Phi(\Omega)$, the function $\varphi^{*}: \Omega \times \mathbb{R}$ defined as

$$
\varphi^{*}(x, s)=\sup _{t \geq 0}(s t-\varphi(x, t)) \text { for a.a. } x \in \Omega \text { and all } s \in[0, \infty)
$$

is called conjugate function of $\varphi$ in the sense of Young.

Proposition 2.7 (cf. [23, Lemma 2.6.5]) Let $\varphi \in \Phi(\Omega)$, then the following Hölder-type inequality holds

$$
\int_{\Omega}|u v| d x \leq 2\|u\|_{\varphi}\|v\|_{\varphi^{*}}, \quad \text { for all } u \in L^{\varphi}(\Omega) \text { and } v \in L^{\varphi^{*}}(\Omega) .
$$

Definition $2.8 \varphi:[0, \infty) \rightarrow[0, \infty)$ is called $N$-function $(N$ stands for nice) if it is a $\Phi$-function satisfying

$$
\lim _{t \rightarrow 0^{+}} \frac{\varphi(t)}{t}=0 \text { and } \lim _{t \rightarrow \infty} \frac{\varphi(t)}{t}=\infty .
$$

A function $\varphi: \Omega \times \mathbb{R} \rightarrow[0, \infty)$ is said to be a generalized $N$-function, and is denoted by $\varphi \in N(\Omega)$, if $\varphi(\cdot, t)$ is measurable for all $t \in \mathbb{R}$ and $\varphi(x, \cdot)$ is an $N$-function for a.a. $x \in \Omega$.

Remark $\varphi \in N(\Omega)$ implies $\varphi^{*} \in N(\Omega)$.

Definition 2.9 Let $\phi, \psi \in N(\Omega)$. We say that $\phi$ increases essentially more slowly than $\psi$ near infinity, and we write $\phi \ll \psi$, if for any $k>0$

$$
\lim _{t \rightarrow \infty} \frac{\phi(x, k t)}{\psi(x, t)}=0 \quad \text { uniformly for a.a. } x \in \Omega \text {. }
$$

For $\varphi \in \Phi(\Omega)$, the related Sobolev space $W^{1, \varphi}(\Omega)$ is the set of all $L^{\varphi}(\Omega)$-functions $u$ having $|\nabla u| \in L^{\varphi}(\Omega)$, and is equipped with the norm

$$
\|u\|_{1, \varphi}=\|u\|_{\varphi}+\|\nabla u\|_{\varphi},
$$

where $\|\nabla u\|_{\varphi}$ stands for $\||\nabla u|\|_{\varphi}$. Furthermore, if $\varphi \in N(\Omega)$ is locally integrable, we denote by $W_{0}^{1, \varphi}(\Omega)$ the completion of $C_{0}^{\infty}(\Omega)$ in $W^{1, \varphi}(\Omega)$.

Proposition 2.10 (cf. [48, Theorem 10.2], [27, Proposition 1.8]) Let $\varphi \in N(\Omega)$ be locally integrable and such that

$$
\inf _{x \in \Omega} \varphi(x, 1)>0 .
$$

Then the spaces $W^{1, \varphi}(\Omega)$ and $W_{0}^{1, \varphi}(\Omega)$ are Banach spaces which are reflexive if $L^{\varphi}(\Omega)$ is reflexive.

\subsection{The double phase $N$-function}

The function $\mathcal{H}: \Omega \times[0, \infty) \rightarrow[0, \infty)$ defined as

$$
\mathcal{H}(x, t):=t^{p}+a(x) t^{q} \text { for all }(x, t) \in \Omega \times[0, \infty),
$$

with $1<p<q$ and $0 \leq a(\cdot) \in L^{1}(\Omega)$, is a locally integrable, generalized $N$-function satisfying (2.2) and

$$
\mathcal{H}(x, 2 t) \leq 2^{q} \mathcal{H}(x, t) \text { for a.a. } x \in \Omega \text { and all } t \in[0, \infty),
$$


that is condition $\left(\Delta_{2}\right)$. Therefore, in correspondence to $\mathcal{H}$, we define the Musielak-Orlicz space $\left(L^{\mathcal{H}}(\Omega),\|\cdot\|_{\mathcal{H}}\right)$ as

$$
\begin{aligned}
L^{\mathcal{H}}(\Omega) & :=\left\{u: \Omega \rightarrow \mathbb{R} \text { measurable }: \varrho_{\mathcal{H}}(u)<\infty\right\}, \\
\|u\|_{\mathcal{H}} & :=\inf \left\{\gamma>0: \varrho_{\mathcal{H}}(u / \gamma) \leq 1\right\},
\end{aligned}
$$

where we recall that

$$
\varrho_{\mathcal{H}}(u):=\int_{\Omega} \mathcal{H}(x,|u|) \mathrm{d} x
$$

Remark 2.11 In the next lemma, we provide an explicit expression for $\|\cdot\|_{\mathcal{H}}$. To this aim, for every function $u$ with $a(x)|u|^{q} \in L^{1}(\Omega)$ and $\left\|a^{1 / q} u\right\|_{q}>0$, we set

$$
\Theta_{p, q}(u):=\left(\frac{\left\|a^{1 / q} u\right\|_{q}}{\|u\|_{p}}\right)^{\frac{q}{q-p}} .
$$

We observe that the convex function

$$
\mathcal{W}(t)=t^{p}+t^{q}, \quad t \in[0, \infty)
$$

is invertible in $[0, \infty)$. We have the following result.

Lemma 2.12 Let $1<p<q$. Then, for every $u$ with $a(x)|u|^{q} \in L^{1}(\Omega)$ and $\left\|a^{1 / q} u\right\|_{q}>0$, there holds

$$
\|u\|_{\mathcal{H}}=\frac{\|u\|_{p} \Theta_{p, q}(u)}{\mathcal{W}^{-1}\left(\Theta_{p, q}(u)^{p}\right)} .
$$

Proof Let $\gamma>0$ be admissible for the problem defining $\|u\|_{\mathcal{H}}$, i.e.,

$$
\frac{1}{\gamma^{p}} \int_{\Omega}|u|^{p} \mathrm{~d} x+\frac{1}{\gamma^{q}} \int_{\Omega} a(x)|u|^{q} \mathrm{~d} x \leq 1 .
$$

We now perform the change of variable

$$
\gamma=\frac{1}{t}\left(\int_{\Omega}|u|^{p} \mathrm{~d} x\right)^{\alpha}\left(\int_{\Omega} a(x)|u|^{q} \mathrm{~d} x\right)^{-\alpha}, \quad t>0,
$$

for some $\alpha \in \mathbb{R}$ that will be chosen later. Then (2.4) becomes

$$
\begin{aligned}
& t^{p}\left(\int_{\Omega}|u|^{p} \mathrm{~d} x\right)^{1-\alpha p}\left(\int_{\Omega} a(x)|u|^{q} \mathrm{~d} x\right)^{\alpha p} \\
& +t^{q}\left(\int_{\Omega}|u|^{p} \mathrm{~d} x\right)^{-\alpha q}\left(\int_{\Omega} a(x)|u|^{q} \mathrm{~d} x\right)^{1+\alpha q} \leq 1 .
\end{aligned}
$$

If we choose

$$
\alpha:=-\frac{1}{q-p}
$$

the previous inequality becomes

$$
\left(t^{p}+t^{q}\right)\left\|a^{1 / q} u\right\|_{q}^{-\frac{p q}{q-p}}\|u\|_{p}^{\frac{p q}{q-p}} \leq 1 .
$$

This can be finally rewritten as

$$
\mathcal{W}(t) \leq \Theta_{p, q}(u)^{p}
$$


This shows that

$$
\|u\|_{\mathcal{H}}=\frac{\left(\int_{\Omega} a(x)|u|^{q} \mathrm{~d} x\right)^{\frac{1}{q-p}}}{\left(\int_{\Omega}|u|^{p} \mathrm{~d} x\right)^{\frac{1}{q-p}}} \inf \left\{\frac{1}{t}>0: \mathcal{W}(t) \leq \Theta_{p, q}(u)^{p}\right\} .
$$

By using that $\mathcal{W}$ is strictly monotonically increasing in $[0, \infty)$, we get the expression (2.3).

We recall here the following definition.

Definition 2.13 A function $\varphi \in N(\Omega)$ is uniformly convex if for every $\varepsilon>0$ there exists $\delta>0$ such that

$$
|t-s| \leq \varepsilon \max \{t, s\} \quad \text { or } \varphi\left(x, \frac{t+s}{2}\right) \leq(1-\delta) \frac{\varphi(x, t)+\varphi(x, s)}{2}
$$

for all $t, s \geq 0$ and a.a. $x \in \Omega$.

We endow the spaces $W^{1, \mathcal{H}}(\Omega)$ and $W_{0}^{1, \mathcal{H}}(\Omega)$ with the norm

$$
\|u\|_{1, \mathcal{H}}:=\|u\|_{\mathcal{H}}+\|\nabla u\|_{\mathcal{H}} .
$$

Proposition 2.14 The spaces $L^{\mathcal{H}}(\Omega), W^{1, \mathcal{H}}(\Omega)$ and $W_{0}^{1, \mathcal{H}}(\Omega)$ are uniformly convex, and so reflexive, Banach spaces.

Proof By Propositions 2.2 and 2.10, $L^{\mathcal{H}}(\Omega), W^{1, \mathcal{H}}(\Omega)$, and $W_{0}^{1, \mathcal{H}}(\Omega)$ are complete. For the second part of the thesis, it suffices to prove that $L^{\mathcal{H}}(\Omega)$ is reflexive. Since by Propostion 2.2, $L^{\mathcal{H}}(\Omega)$ is a Banach space, if we prove that $L^{\mathcal{H}}(\Omega)$ is uniformly convex, the reflexivity follows by the Milman-Pettis theorem. By Theorems 2.4.11 and 2.4.14 of [23], in order to prove that $L^{\mathcal{H}}(\Omega)$ is uniformly convex, it is enough to show that the $N$-function $\mathcal{H}$ is uniformly convex. Let $\varepsilon>0$ and $t, s \geq 0$ be such that $|t-s|>\varepsilon \max \{t, s\}$. By Remark 2.4.16 of [23] there exist $\delta_{p}(\varepsilon), \delta_{q}(\varepsilon)>0$ such that

$$
\left(\frac{t+s}{2}\right)^{p} \leq\left(1-\delta_{p}(\varepsilon)\right) \frac{t^{p}+s^{p}}{2} \text { and }\left(\frac{t+s}{2}\right)^{q} \leq\left(1-\delta_{q}(\varepsilon)\right) \frac{t^{q}+s^{q}}{2},
$$

thus

$$
\left(\frac{t+s}{2}\right)^{p}+a(x)\left(\frac{t+s}{2}\right)^{q} \leq\left(1-\min \left\{\delta_{p}(\varepsilon), \delta_{q}(\varepsilon)\right\}\right) \frac{t^{p}+a(x) t^{q}+s^{p}+a(x) s^{q}}{2} .
$$

This concludes the proof.

In the following, the notation $X \hookrightarrow Y$ means that the space $X$ is continuously embedded into the space $Y$, while $X \hookrightarrow \hookrightarrow Y$ means that $X$ is compactly embedded into $Y$.

Proposition 2.15 (Embeddings, I) Put $p^{*}:=n p /(n-p)$ if $p<n, p^{*}:=+\infty$ otherwise, and

$$
L_{a}^{q}(\Omega):=\left\{u: \Omega \rightarrow \mathbb{R} \text { measurable: } \int_{\Omega} a(x)|u|^{q} \mathrm{~d} x<\infty\right\},
$$

endowed with the norm

$$
\|u\|_{q, a}:=\left(\int_{\Omega} a(x)|u|^{q} \mathrm{~d} x\right)^{1 / q} .
$$

Then the following embeddings hold: 
(i) $L^{\mathcal{H}}(\Omega) \hookrightarrow L^{r}(\Omega)$ and $W_{0}^{1, \mathcal{H}}(\Omega) \hookrightarrow W_{0}^{1, r}(\Omega)$ for all $r \in[1, p]$;

(ii) if $p \neq n$, then

$$
W_{0}^{1, \mathcal{H}}(\Omega) \hookrightarrow L^{r}(\Omega) \text { for all } r \in\left[1, p^{*}\right]
$$

if $p=n$, then

$$
W_{0}^{1, \mathcal{H}}(\Omega) \hookrightarrow L^{r}(\Omega) \text { for all } r \in[1, \infty)
$$

(iii) if $p \leq n$, then

$$
W_{0}^{1, \mathcal{H}}(\Omega) \hookrightarrow \hookrightarrow L^{r}(\Omega) \text { for all } r \in\left[1, p^{*}\right) ;
$$

if $p>n$, then

$$
W_{0}^{1, \mathcal{H}}(\Omega) \hookrightarrow \hookrightarrow L^{\infty}(\Omega)
$$

(iv) $L^{\mathcal{H}}(\Omega) \hookrightarrow L_{a}^{q}(\Omega)$;

(v) if $a \in L^{\infty}(\Omega)$, then $L^{q}(\Omega) \hookrightarrow L^{\mathcal{H}}(\Omega)$.

Proof Put $\mathcal{H}_{p}(x, t):=t^{p}$ for all $t \geq 0$ and $x \in \Omega$. Clearly, $\mathcal{H}_{p} \preceq \mathcal{H}$, hence by Proposition 2.3, $L^{\mathcal{H}}(\Omega) \hookrightarrow L^{p}(\Omega)$ and $W_{0}^{1, \mathcal{H}}(\Omega) \hookrightarrow W_{0}^{1, p}(\Omega)$. Therefore, $(i)$ follows by the boundedness of $\Omega$. While, (ii) and (iii) follow by the embedding results on classical Lebesgue and Sobolev spaces. Now, let $u \in L^{\mathcal{H}}(\Omega)$, then

$$
\int_{\Omega} a(x)|u|^{q} \mathrm{~d} x \leq \int_{\Omega}\left(|u|^{p}+a(x)|u|^{q}\right) \mathrm{d} x=\varrho_{\mathcal{H}}(u) .
$$

Therefore, if $u \neq 0$,

$$
\int_{\Omega} a(x)\left(\frac{|u|}{\|u\|_{\mathcal{H}}}\right)^{q} \mathrm{~d} x \leq 1,
$$

and so we conclude

$$
\|u\|_{q, a} \leq\|u\|_{\mathcal{H}},
$$

which proves (iv). Finally, for all $t \geq 0$ and a.a. $x \in \Omega$, if $a \in L^{\infty}(\Omega)$, we have

$$
\mathcal{H}(x, t) \leq\left(1+t^{q}\right)+a(x) t^{q} \leq 1+\left(1+\|a\|_{\infty}\right) t^{q},
$$

so $(v)$ follows once again by Proposition 2.3.

Although we will not use it explicitly, the next lemma could be useful in some situations.

Lemma 2.16 Let $\left(u_{h}\right)$ be a sequence in $L^{\mathcal{H}}(\Omega)$ such that $u_{h} \rightarrow u \in L^{\mathcal{H}}(\Omega)$. Then, there exist a subsequence $\left(u_{h_{j}}\right)$ and a function $v \in L^{\mathcal{H}}(\Omega)$ such that

$$
\begin{aligned}
& u_{h_{j}} \rightarrow u \text { a.e. in } \Omega, \\
& \left|u_{h_{j}}\right| \leq v \text { for all } j, \text { a.e. in } \Omega .
\end{aligned}
$$

Proof By Proposition 2.15 $L^{\mathcal{H}}(\Omega) \hookrightarrow L^{p}(\Omega)$, hence up to a subsequence $u_{h} \rightarrow u$ a.e. in $\Omega$. We prove now the second part of the statement. Since $\left(u_{h}\right)$ is a Cauchy sequence in $L^{\mathcal{H}}(\Omega)$, we can find a subsequence $\left(u_{h_{j}}\right)$ for which $\left\|u_{h_{j+1}}-u_{h_{j}}\right\|_{\mathcal{H}} \leq 2^{-j}$. For all $m \geq 1$ we define

$$
f_{m}(x):=\sum_{j=1}^{m}\left|u_{h_{j+1}}(x)-u_{h_{j}}(x)\right|
$$


which satisfies

$$
\left\|f_{m}\right\|_{\mathcal{H}} \leq \sum_{j=1}^{m}\left\|u_{h_{j+1}}-u_{h_{j}}\right\|_{\mathcal{H}} \leq 1 \text { for all } m \in \mathbb{N}
$$

and by the unit ball property

$$
\varrho_{\mathcal{H}}\left(f_{m}\right) \leq 1 \text { for all } m \in \mathbb{N} \text {. }
$$

Clearly, $f_{m}(x) \leq f_{m+1}(x)$ for all $m$, a.e. in $\Omega$, and so, by the monotone convergence theorem, there exists $f \in L^{1}(\Omega)$ such that $f_{m} \rightarrow f$ a.e. in $\Omega$. By virtue of Lemma 2.3.16-(b) of [23],

$$
\varrho_{\mathcal{H}}(f)=\lim _{m \rightarrow \infty} \varrho_{\mathcal{H}}\left(f_{m}\right),
$$

whence $f \in L^{\mathcal{H}}(\Omega)$. Now, for all $m>\ell \geq 2$ and for a.a. $x \in \Omega$

$$
\begin{aligned}
& \left|u_{h_{m}}(x)-u_{h_{\ell}}(x)\right| \leq\left|u_{h_{m}}(x)-u_{h_{m-1}}(x)\right|+\cdots+\left|u_{h_{\ell+1}}(x)-u_{h_{\ell}}(x)\right| \\
& \quad=f_{m-1}(x)-f_{\ell-1}(x) .
\end{aligned}
$$

This implies that for a.a. $x \in \Omega,\left(u_{h_{j}}(x)\right)$ is a Cauchy sequence in $\mathbb{R}$ and so it converges to some $\bar{u}(x) \in \mathbb{R}$. Furthermore, passing to the limit for $m \rightarrow \infty$ in (2.5), we get for all $j \geq 2$ and for a.a. $x \in \Omega$

$$
\left|\bar{u}(x)-u_{h_{j}}(x)\right| \leq f(x) .
$$

Thus, by Lemma 2.3.16 (c) of [23], we obtain $u_{h_{j}} \rightarrow \bar{u}$ in $L^{\mathcal{H}}(\Omega)$, and so $u=\bar{u}$ a.e. in $\Omega$. Finally, (2.6) yields

$$
\left|u_{h_{j}}(x)\right| \leq|u(x)|+f(x) \text { for a.a. } x \in \Omega,
$$

and the proof is concluded by taking $v:=|u|+f \in L^{\mathcal{H}}(\Omega)$.

From now on in the paper, unless explicitly stated, we shall assume that

$$
1<p<q<n \text {. }
$$

Definition 2.17 For all $x \in \Omega$ denote by $\mathcal{H}^{-1}(x, \cdot):[0, \infty) \rightarrow[0, \infty)$ the inverse function of $\mathcal{H}(x, \cdot)$ and define $\mathcal{H}_{*}^{-1}: \Omega \times[0, \infty) \rightarrow[0, \infty)$ by

$$
\mathcal{H}_{*}^{-1}(x, s)=\int_{0}^{s} \frac{\mathcal{H}^{-1}(x, \tau)}{\tau^{(n+1) / n}} d \tau \text { for all }(x, s) \in \Omega \times[0, \infty) .
$$

The function $\mathcal{H}_{*}:(x, t) \in \Omega \times[0, \infty) \mapsto s \in[0, \infty)$ such that $\mathcal{H}_{*}^{-1}(x, s)=t$ is called Sobolev conjugate function of $\mathcal{H}$.

Proposition 2.18 (Embeddings, II) Assume that (1.7) holds. Then the following facts hold.

(i) $W^{1, \mathcal{H}}(\Omega) \hookrightarrow L^{\mathcal{H}_{*}}(\Omega)$.

(ii) If $\mathcal{K} \in N(\Omega), \mathcal{K}: \Omega \times[0, \infty) \rightarrow[0, \infty)$ is continuous and such that $\mathcal{K} \ll \mathcal{H}_{*}$, then

$$
W^{1, \mathcal{H}}(\Omega) \hookrightarrow \hookrightarrow L^{\mathcal{K}}(\Omega) .
$$

(iii) $\mathcal{H} \ll \mathcal{H}_{*}$, and consequently

$$
W^{1, \mathcal{H}}(\Omega) \hookrightarrow \hookrightarrow L^{\mathcal{H}}(\Omega) .
$$


(iv) The following Poincaré-type inequality holds

$$
\|u\|_{\mathcal{H}} \leq C\|\nabla u\|_{\mathcal{H}}, \quad \text { for all } u \in W_{0}^{1, \mathcal{H}}(\Omega),
$$

for some constant $C>0$ independent of $u$.

Proof We refer to Theorems 1.1 and 1.2 of [26]. It suffices to prove condition (2) of Proposition 3.1 of [26], i.e., that there exist three positive constants $\delta<1 / n, c_{0}$ and $t_{0}$ such that

$$
\left|\frac{\partial \mathcal{H}(x, t)}{\partial x_{j}}\right| \leq c_{0}(\mathcal{H}(x, t))^{1+\delta}
$$

for all $j=1, \ldots, n, x \in \Omega$ for which $\nabla a(x)$ exists, and $t \geq t_{0}$. If we put $\delta:=q / p-1$ and $c_{a}>0$ the Lipschitz constant of $a$, we get

$$
\left|\frac{\partial \mathcal{H}(x, t)}{\partial x_{j}}\right| \leq c_{a} t^{q} \leq c_{a}\left(t^{p}+a(x) t^{q}\right)^{q / p} \text { for a.a. } x \in \Omega \text {, all } t>0 \text {, and } j=1, \ldots, n,
$$

that is (2.8) with $c_{0}:=c_{a}, \delta:=q / p-1<1 / n$ and any $t_{0}>0$.

Remark 2.19 Poincaré-type inequality (2.7) has been proved also in [32] under the more general assumption

$$
\Omega \text { is quasiconvex and } a \in C^{0, \alpha}(\bar{\Omega}) \text {, with } \frac{q}{p} \leq 1+\frac{\alpha}{n} \text { for some } \alpha \in(0,1] \text {. }
$$

We wish to stress that the bound on $q$ / $p$ given in (2.9) was required for the first time in the papers $[3,5]$ dealing with regularity of local minimizers for double phase variational integrals. Furthermore, we observe that, since $p^{*}>p(1+1 / n)$, both (1.7) and (2.9) imply $q<p^{*}$.

As a consequence of inequality (2.7), if either assumption (1.7) or assumption (2.9) holds, we equip the space $W_{0}^{1, \mathcal{H}}(\Omega)$ with the equivalent norm

$$
\|\nabla u\|_{\mathcal{H}} .
$$

\section{The eigenvalue problem}

\subsection{Derivation of the Euler-Lagrange equation}

Put

$K(u):=\|\nabla u\|_{\mathcal{H}}, \quad k(u):=\|u\|_{\mathcal{H}}$ for $u \in W_{0}^{1, \mathcal{H}}(\Omega), \quad \mathcal{M}:=\left\{u \in W_{0}^{1, \mathcal{H}}(\Omega): k(u)=1\right\}$.

Let us consider the Rayleigh ratio

$$
\frac{K(u)}{k(u)}=\frac{\|\nabla u\|_{\mathcal{H}}}{\|u\|_{\mathcal{H}}}
$$

and define the first eigenvalue as

$$
\lambda_{\mathcal{H}}^{1}:=\inf _{u \in W_{0}^{1, \mathcal{H}}(\Omega) \backslash\{0\}} \frac{\|\nabla u\|_{\mathcal{H}}}{\|u\|_{\mathcal{H}}}=\inf _{u \in \mathcal{M}} K(u) .
$$


We claim that the following equation

$$
\begin{aligned}
-\operatorname{div}( & {\left.\left[p\left(\frac{|\nabla u|}{K(u)}\right)^{p-2}+q a(x)\left(\frac{|\nabla u|}{K(u)}\right)^{q-2}\right] \frac{\nabla u}{K(u)}\right) } \\
& =\lambda S(u)\left[p\left(\frac{|u|}{k(u)}\right)^{p-2}+q a(x)\left(\frac{|u|}{k(u)}\right)^{q-2}\right] \frac{u}{k(u)}, \quad u \in W_{0}^{1, \mathcal{H}}(\Omega) \backslash\{0\}
\end{aligned}
$$

is the Euler-Lagrange equation corresponding to the minimization of the Rayleigh ratio (3.1). In (3.3), we have denoted by $S(u)$ the following quantity

$$
S(u):=\frac{\int_{\Omega}\left[p\left(\frac{|\nabla u|}{K(u)}\right)^{p}+q a(x)\left(\frac{|\nabla u|}{K(u)}\right)^{q}\right] \mathrm{d} x}{\int_{\Omega}\left[p\left(\frac{|u|}{k(u)}\right)^{p}+q a(x)\left(\frac{|u|}{k(u)}\right)^{q}\right] \mathrm{d} x} .
$$

We note that Eq. (3.3) reduces to (1.8) if $u \in \mathcal{M}$, since in that case $K(u)=\lambda, k(u)=1$ and $S(u)$ reads as in (1.9).

In order to prove the claim, we define for all $u \in W_{0}^{1, \mathcal{H}}(\Omega) \backslash\{0\}$ and $v \in W_{0}^{1, \mathcal{H}}(\Omega)$ :

$$
\begin{aligned}
& \langle A(u), v\rangle:=\frac{\int_{\Omega}\left[p\left(\frac{|u|}{k(u)}\right)^{p-2}+q a(x)\left(\frac{|u|}{k(u)}\right)^{q-2}\right] \frac{u}{k(u)} v \mathrm{~d} x}{\int_{\Omega}\left[p\left(\frac{|u|}{k(u)}\right)^{p}+q a(x)\left(\frac{|u|}{k(u)}\right)^{q}\right] \mathrm{d} x}, \\
& \langle B(u), v\rangle:=\frac{\int_{\Omega}\left[p\left(\frac{|\nabla u|}{K(u)}\right)^{p-2}+q a(x)\left(\frac{|\nabla u|}{K(u)}\right)^{q-2}\right] \frac{\nabla u}{K(u)} \cdot \nabla v \mathrm{~d} x}{\int_{\Omega}\left[p\left(\frac{|\nabla u|}{K(u)}\right)^{p}+q a(x)\left(\frac{|\nabla u|}{K(u)}\right)^{q}\right] \mathrm{d} x} .
\end{aligned}
$$

Proposition 3.1 $k \in C^{1}\left(W_{0}^{1, \mathcal{H}}(\Omega) \backslash\{0\}\right)$ with $k^{\prime}(u)=A(u)$ for all $u \in W_{0}^{1, \mathcal{H}}(\Omega) \backslash\{0\}$.

Proof Reasoning as in Lemma A.1 of [28], we get

$$
\lim _{\varepsilon \rightarrow 0^{+}} \frac{k(u+\varepsilon v)-k(u)}{\varepsilon}=\langle A(u), v\rangle .
$$

Put

$$
f(v):=\int_{\Omega}\left[p\left(\frac{|v|}{k(v)}\right)^{p}+q a(x)\left(\frac{|v|}{k(v)}\right)^{q}\right] \mathrm{d} x \text { for all } v \in W_{0}^{1, \mathcal{H}}(\Omega) \backslash\{0\} .
$$

We observe that, being $1<p<q$ and by the unit ball property,

$$
f(v) \geq \varrho_{\mathcal{H}}\left(\frac{v}{k(v)}\right)=1 .
$$

By Hölder's inequality and by Proposition 2.15, it results that

$$
\begin{aligned}
|\langle A(u), v\rangle| & \leq \frac{p}{k(u)^{p-1}} \int_{\Omega}|u|^{p-1}|v| \mathrm{d} x+\frac{q}{k(u)^{q-1}} \int_{\Omega} a^{1 / q^{\prime}}|u|^{q-1} a^{1 / q}|v| \mathrm{d} x \\
& \leq \frac{p}{k(u)^{p-1}}\|u\|_{p}^{p-1}\|v\|_{p}+\frac{q}{k(u)^{q-1}}\|u\|_{q, a}^{q-1}\|v\|_{q, a} \\
& \leq\left(\frac{\mathcal{S}_{p} p}{k(u)^{p-1}}\|u\|_{p}^{p-1}+\frac{\mathcal{S}_{q, a} q}{k(u)^{q-1}}\|u\|_{q, a}^{q-1}\right)\|v\|_{1, \mathcal{H}},
\end{aligned}
$$


where $\mathcal{S}_{p}, \mathcal{S}_{q, a}>0$ are the Sobolev constants for the embeddings of $W_{0}^{1, \mathcal{H}}(\Omega)$ in $L^{p}(\Omega)$ and in $L_{a}^{q}(\Omega)$, respectively. Therefore, $A(u)$ belongs to the dual space $\left(W_{0}^{1, \mathcal{H}}(\Omega)\right)^{\prime}$ of $W_{0}^{1, \mathcal{H}}(\Omega)$ and $k$ is Gâteaux differentiable in $u$, with $k^{\prime}(u)=A(u)$ for all $u \in W_{0}^{1, \mathcal{H}}(\Omega) \backslash\{0\}$. It remains to prove that $k^{\prime}: W_{0}^{1, \mathcal{H}}(\Omega) \rightarrow\left(W_{0}^{1, \mathcal{H}}(\Omega)\right)^{\prime}$ is continuous, i.e., that given a sequence $\left(u_{h}\right) \subset W_{0}^{1, \mathcal{H}}(\Omega) \backslash\{0\}$ such that $u_{h} \rightarrow u \neq 0$ in $W_{0}^{1, \mathcal{H}}(\Omega)$,

$$
\sup _{\substack{v \in W_{0}^{1, \mathcal{H}}(\Omega) \\\|v\|_{1, \mathcal{H}} \leq 1}}\left|\left\langle k^{\prime}\left(u_{h}\right)-k^{\prime}(u), v\right\rangle\right| \rightarrow 0 \quad \text { as } h \rightarrow \infty .
$$

For all $v \in W_{0}^{1, \mathcal{H}}(\Omega)$ with $\|v\|_{1, \mathcal{H}} \leq 1$ we get

$$
\begin{aligned}
& \left|\left\langle k^{\prime}\left(u_{h}\right)-k^{\prime}(u), v\right\rangle\right| \\
& \leq\left|\frac{\int_{\Omega} p\left(\frac{\left|u_{h}\right|}{k\left(u_{h}\right)}\right)^{p-2} \frac{u_{h}}{k\left(u_{h}\right)} v \mathrm{~d} x}{f\left(u_{h}\right)}-\frac{\int_{\Omega} p\left(\frac{|u|}{k(u)}\right)^{p-2} \frac{u}{k(u)} v \mathrm{~d} x}{f(u)}\right| \\
& +\left|\frac{\int_{\Omega} q a(x)\left(\frac{\left|u_{h}\right|}{k\left(u_{h}\right)}\right)^{q-2} \frac{u_{h}}{k\left(u_{h}\right)} v \mathrm{~d} x}{f\left(u_{h}\right)}-\frac{\int_{\Omega} q a(x)\left(\frac{|u|}{k(u)}\right)^{q-2} \frac{u}{k(u)} v \mathrm{~d} x}{f(u)}\right| \\
& \leq p I_{1}^{(h)}+q I_{2}^{(h)},
\end{aligned}
$$

where

$$
\begin{aligned}
I_{1}^{(h)} & :=\int_{\Omega}|v|\left|\frac{1}{f\left(u_{h}\right)}\left(\frac{\left|u_{h}\right|}{k\left(u_{h}\right)}\right)^{p-2} \frac{u_{h}}{k\left(u_{h}\right)}-\frac{1}{f(u)}\left(\frac{|u|}{k(u)}\right)^{p-2} \frac{u}{k(u)}\right| \mathrm{d} x \\
I_{2}^{(h)} & :=\int_{\Omega} a(x)|v|\left|\frac{1}{f\left(u_{h}\right)}\left(\frac{\left|u_{h}\right|}{k\left(u_{h}\right)}\right)^{q-2} \frac{u_{h}}{k\left(u_{h}\right)}-\frac{1}{f(u)}\left(\frac{|u|}{k(u)}\right)^{q-2} \frac{u}{k(u)}\right| \mathrm{d} x .
\end{aligned}
$$

We show that $I_{2}^{(h)} \rightarrow 0$ as $h \rightarrow \infty$, the proof for $I_{1}^{(h)}$ is identical, with $a(\cdot)$ replaced by the constant function 1 and $q$ replaced by $p$. We get by Hölder's inequality

$$
\begin{aligned}
I_{2}^{(h)} \leq & \int_{\Omega} \frac{a(x)^{1 / q}|v|}{f\left(u_{h}\right)} a(x)^{1 / q^{\prime}}\left|\left(\frac{\left|u_{h}\right|}{k\left(u_{h}\right)}\right)^{q-2} \frac{\left|u_{h}\right|}{k\left(u_{h}\right)}-\left(\frac{|u|}{k(u)}\right)^{q-2} \frac{|u|}{k(u)}\right| \mathrm{d} x \\
& +\int_{\Omega} a(x)^{1 / q}|v| a(x)^{1 / q^{\prime}}\left(\frac{|u|}{k(u)}\right)^{q-1}\left|\frac{1}{f\left(u_{h}\right)}-\frac{1}{f(u)}\right| \mathrm{d} x \\
\leq & \|v\|_{q, a}\left(\int_{\Omega} a(x)\left|\left(\frac{\left|u_{h}\right|}{k\left(u_{h}\right)}\right)^{q-2} \frac{\left|u_{h}\right|}{k\left(u_{h}\right)}-\left(\frac{|u|}{k(u)}\right)^{q-2} \frac{|u|}{k(u)}\right|^{q^{\prime}} \mathrm{d} x\right)^{1 / q^{\prime}} \\
& +\|v\|_{q, a}\left|\frac{1}{f\left(u_{h}\right)}-\frac{1}{f(u)}\right|\left(\int_{\Omega} a(x)\left(\frac{|u|}{k(u)}\right)^{q} \mathrm{~d} x\right)^{1 / q^{\prime}} \\
\leq & \mathcal{S}_{q, a}\left(\mathcal{I}^{(h)}+\mathcal{J}^{(h)}\right),
\end{aligned}
$$


where

$$
\begin{aligned}
\mathcal{I}^{(h)} & :=\left(\int_{\Omega} a(x)\left|\left(\frac{\left|u_{h}\right|}{k\left(u_{h}\right)}\right)^{q-2} \frac{\left|u_{h}\right|}{k\left(u_{h}\right)}-\left(\frac{|u|}{k(u)}\right)^{q-2} \frac{|u|}{k(u)}\right|^{q^{\prime}} \mathrm{d} x\right)^{1 / q^{\prime}} \\
\mathcal{J}^{(h)} & :=\left|\frac{1}{f\left(u_{h}\right)}-\frac{1}{f(u)}\right|\left(\int_{\Omega} a(x)\left(\frac{|u|}{k(u)}\right)^{q} \mathrm{~d} x\right)^{1 / q^{\prime}} .
\end{aligned}
$$

First, we estimate $\mathcal{I}^{(h)}$. We pick a subsequence $\left(u_{h_{j}}\right)$. Since by Proposition $2.15 W_{0}^{1, \mathcal{H}}(\Omega) \hookrightarrow$ $L_{a}^{q}(\Omega)$, thus $u_{h_{j}} \rightarrow u$ in $L_{a}^{q}(\Omega)$, i.e., $a^{1 / q} u_{h_{j}} \rightarrow a^{1 / q} u$ in $L^{q}(\Omega)$. Up to a subsequence, $u_{h_{j}} \rightarrow u$ a.e. in $\Omega$ and there exists a function $w \in L^{q}(\Omega)$ such that $a^{1 / q}\left|u_{h_{j}}\right| \leq w$ a.e. in $\Omega$ for all $j$. Therefore, for $j$ sufficiently large and for $\varepsilon \in(0, k(u))$,

$$
\begin{aligned}
& a(x)\left|\left(\frac{\left|u_{h_{j}}\right|}{k\left(u_{h_{j}}\right)}\right)^{q-2} \frac{u_{h_{j}}}{k\left(u_{h_{j}}\right)}-\left(\frac{|u|}{k(u)}\right)^{q-2} \frac{u}{k(u)}\right|^{q^{\prime}} \\
& \leq 2^{q^{\prime}-1} a(x)\left[\left(\frac{\left|u_{h_{j}}\right|}{k\left(u_{h_{j}}\right)}\right)^{q}+\left(\frac{|u|}{k(u)}\right)^{q}\right] \\
& \leq 2^{q^{\prime}-1}\left[\left(\frac{w}{k\left(u_{h_{j}}\right)}\right)^{q}+\left(\frac{w}{k(u)}\right)^{q}\right] \\
& \leq 2^{q^{\prime}-1}\left[\frac{1}{(k(u)-\varepsilon)^{q}}+\frac{1}{k(u)^{q}}\right] w^{q} \in L^{1}(\Omega),
\end{aligned}
$$

where in the last inequality we used the fact that $k\left(u_{h_{j}}\right) \rightarrow k(u) \neq 0$. Consequently, by the dominated convergence theorem, $\mathcal{I}^{\left(h_{j}\right)} \rightarrow 0$ as $j \rightarrow \infty$ and by the arbitrariness of the subsequence, $\mathcal{I}^{(h)} \rightarrow 0$ as $h \rightarrow \infty$. Now, in order to prove that also $\mathcal{J}^{(h)} \rightarrow 0$, it is enough to prove that $f\left(u_{h}\right) \rightarrow f(u)$ as $h \rightarrow \infty$. By the facts that $W_{0}^{1, \mathcal{H}}(\Omega) \hookrightarrow L^{p}(\Omega)$ and $W_{0}^{1, \mathcal{H}}(\Omega) \hookrightarrow L_{a}^{q}(\Omega)$, and by the dominated convergence theorem, we easily get

$$
\begin{aligned}
& \lim _{h \rightarrow \infty} \int_{\Omega}\left(\frac{\left|u_{h}\right|}{k\left(u_{h}\right)}\right)^{p} \mathrm{~d} x=\int_{\Omega}\left(\frac{|u|}{k(u)}\right)^{p} \mathrm{~d} x \\
& \lim _{h \rightarrow \infty} \int_{\Omega} a(x)\left(\frac{\left|u_{h}\right|}{k\left(u_{h}\right)}\right)^{q} \mathrm{~d} x=\int_{\Omega} a(x)\left(\frac{|u|}{k(u)}\right)^{q} \mathrm{~d} x .
\end{aligned}
$$

This proves that $f\left(u_{h}\right) \rightarrow f(u)$ and so $\mathcal{J}^{(h)} \rightarrow 0$. Therefore, $\left(I_{2}^{(h)}\right)$ converges to zero as $h \rightarrow \infty$ and the proof is concluded by the arbitrariness of $v$.

As a consequence of the last proposition, $\mathcal{M}$ is a $C^{1}$ Banach manifold. By using analogous techniques as in the proof of Proposition 3.1, it is possible to prove the following result.

Proposition $3.2 K \in C^{1}\left(W_{0}^{1, \mathcal{H}}(\Omega) \backslash\{0\}\right)$ with $K^{\prime}(u)=B(u)$ for all $u \in W_{0}^{1, \mathcal{H}}(\Omega) \backslash\{0\}$.

Reasoning as in Section 3 of [28], we find that a necessary condition for minimality of the Rayleigh ratio (3.1) is

$$
\frac{\left\langle K^{\prime}(u), v\right\rangle}{K(u)}=\frac{\left\langle k^{\prime}(u), v\right\rangle}{k(u)} \text { for all } u, v \in W_{0}^{1, \mathcal{H}}(\Omega), u \neq 0 .
$$

Together with Propositions 3.1 and 3.2, this yields the claim with $\lambda=K(u) / k(u)$, and justifies the following definition. 
Definition 3.3 We say that $u \in W_{0}^{1, \mathcal{H}}(\Omega) \backslash\{0\}$ is an eigenfunction of (3.3) if

$$
\begin{aligned}
& \int_{\Omega}\left(p\left|\frac{\nabla u}{K(u)}\right|^{p-2}+q a(x)\left|\frac{\nabla u}{K(u)}\right|^{q-2}\right) \frac{\nabla u}{K(u)} \cdot \nabla v \mathrm{~d} x \\
& =\lambda S(u) \int_{\Omega}\left(p\left|\frac{u}{k(u)}\right|^{p-2}+q a(x)\left|\frac{u}{k(u)}\right|^{q-2}\right) \frac{u}{k(u)} v \mathrm{~d} x
\end{aligned}
$$

for all $v \in C_{0}^{\infty}(\Omega)$, where $S(u)$ is defined as in (3.4). The real number $\lambda$ is the corresponding eigenvalue. The set

$$
\Lambda:=\{\lambda \in \mathbb{R}: \lambda \text { is eigenvalue of (3.3) }\},
$$

is called spectrum.

We remark here that, by a standard density argument, we can take any $v \in W_{0}^{1, \mathcal{H}}(\Omega)$ as test function in (3.6). Testing Eq. (3.6) with $v=u$ yields

$$
\lambda=\frac{K(u)}{k(u)},
$$

so that if $u \in \mathcal{M}$, then $\lambda=\|\nabla u\|_{\mathcal{H}}$ and Eq. (1.8) holds.

\section{$3.2 L^{\infty}$-bound of eigenfunctions}

Assume that condition (1.7) on $p, q, a$ and $\Omega$ holds. If $u \in \mathcal{M}$ is a weak solution to the Euler-Lagrange equation

$$
-\operatorname{div}\left(p\left|\frac{\nabla u}{\lambda}\right|^{p-2} \frac{\nabla u}{\lambda}+q a(x)\left|\frac{\nabla u}{\lambda}\right|^{q-2} \frac{\nabla u}{\lambda}\right)=\lambda S(u)\left(p|u|^{p-2} u+q a(x)|u|^{q-2} u\right),
$$

with $\lambda=\|\nabla u\|_{\mathcal{H}}>0$ and $S(u)$ is as in formula (1.9), then by following a standard argument it is possible to prove that there exists a positive constant $C(n, p, \lambda, a)$ such that

$$
\|u\|_{L^{\infty}(\Omega)} \leq C(n, p, \lambda, a) .
$$

Observe that $S(u) \leq q$ from formula (1.9) and $\lambda=\|\nabla u\|_{\mathcal{H}}$. For all $t \geq 0$ and $k>0$, we set $t_{k}:=\min \{t, k\}$. For all $r \geq 2, k>0$, the mapping $t \mapsto t|t|_{k}^{r-2}$ is Lipschitz continuous in $\mathbb{R}$, hence $v=u|u|_{k}^{r-2} \in W_{0}^{1, \mathcal{H}}(\Omega)$ and we have

$$
\nabla v=(r-1)|u|_{k}^{r-2} \nabla u, \quad \text { if }|u| \leq k, \quad \nabla v=|u|_{k}^{r-2} \nabla u, \quad \text { if }|u| \geq k .
$$

We choose it as a test function, getting (recall that $|s|_{k} \leq|s|$ for any $s$ and $k$ ),

$$
\begin{aligned}
(r-1) & \int_{\Omega}\left(p\left|\frac{\nabla u}{\lambda}\right|^{p}|u|_{k}^{r-2}+q a(x)\left|\frac{\nabla u}{\lambda}\right|^{q}|u|_{k}^{r-2}\right) \chi_{\{|u| \leq k\}} \mathrm{d} x \\
& \leq S(u) \int_{\Omega}\left(p|u|^{p}+q a(x)|u|^{q}\right)|u|_{k}^{r-2} \mathrm{~d} x \\
& \leq C^{\prime} \int_{\Omega}\left(|u|^{p+r-2}+|u|^{q+r-2}\right) \mathrm{d} x
\end{aligned}
$$


for some positive constant $C^{\prime}$ which depends only on $p, q$, $a$. Taking into account that $a \geq 0$ and using Fatou's lemma (by letting $k \rightarrow \infty$ ), it follows that

$$
(r-1) \int_{\Omega}|\nabla u|^{p}|u|^{r-2} \mathrm{~d} x \leq C \int_{\Omega}\left(|u|^{p+r-2}+|u|^{q+r-2}\right) \mathrm{d} x,
$$

with $C$ depending on $p, q, a$ and $\lambda$. In light of condition (1.7), we know that $q<p^{*}$. But then this is exactly the estimate that is usually obtained to get the $L^{m}$-estimate for any $m \geq 1$ for the $p$-Laplacian problem $-\Delta_{p} u=f(u)$ in $\Omega$ and $u=0$ on $\partial \Omega$, for a subcritical nonlinearity $f: \mathbb{R} \rightarrow \mathbb{R}$ which satisfies the growth condition

$$
|f(s)| \leq C|s|^{p-1}+C|s|^{q-1} \text { for all } s \in \mathbb{R}, \quad 1<q<p^{*} .
$$

For the explicit computations following inequality (3.10) and the bootstrap argument yielding $u \in L^{m}(\Omega)$ for every $m \geq 1$, one can argue, e.g., as in [59]. Then, similar bootstrap arguments allow to prove the $L^{\infty}$ - estimate.

\subsection{On the first eigenvalue}

Throughout this subsection, we shall assume the validity of condition (1.7) and we endow $W_{0}^{1, \mathcal{H}}(\Omega)$ with the $L^{\mathcal{H}}$-norm of the gradient. First of all, we seek ground states, i.e., least energy solutions, of (3.3). In particular, the ground states of (3.3) are the minimizers of $\left.K\right|_{\mathcal{M}}$ and the corresponding energy level is the first eigenvalue $\lambda_{\mathcal{H}}^{1}$. Minimizers $u$, if they exist, must satisfy the Euler-Lagrange Eq. (3.6), obtained for minimizers of the quotients $\|\nabla v\|_{\mathcal{H}} /\|v\|_{\mathcal{H}}$ among nonzero functions. Since $u \in \mathcal{M}$, then Eq. (3.6) can be reduced to (1.8) since it turns out that $\|\nabla u\|_{\mathcal{H}}=\lambda$ by formula (3.7). After giving the proof of Theorem 1.1, we shall collect some properties of the first eigenvalue.

- Proof of Theorem 1.1. By the Poincaré-type inequality (2.7), there exists $C>0$ independent of $v$ for which

$$
\frac{\|\nabla v\|_{\mathcal{H}}}{\|v\|_{\mathcal{H}}} \geq \frac{1}{C}
$$

for all $v \in W_{0}^{1, \mathcal{H}}(\Omega) \backslash\{0\}$. Thus $\lambda_{\mathcal{H}}^{1}>0$. Now, let $\left(v_{h}\right) \subset \mathcal{M}$ be such that

$$
\lim _{h \rightarrow \infty}\left\|\nabla v_{h}\right\|_{\mathcal{H}}=\lambda_{\mathcal{H}}^{1}
$$

Clearly, $\left(v_{h}\right)$ is bounded in the reflexive Banach space $W_{0}^{1, \mathcal{H}}(\Omega)$ and so we can extract a subsequence $\left(v_{h_{j}}\right)$ weakly converging to $u$ in $W_{0}^{1, \mathcal{H}}(\Omega)$. Therefore, by Proposition 2.18(iii), $\left\|v_{h_{j}}\right\|_{\mathcal{H}} \rightarrow\|u\|_{\mathcal{H}}$ as $j \rightarrow \infty$, and so $\|u\|_{\mathcal{H}}=1$. Since the norm is weakly lower semicontinuous,

$$
\|\nabla u\|_{\mathcal{H}} \leq \liminf _{j \rightarrow \infty}\left\|\nabla v_{h_{j}}\right\|_{\mathcal{H}}=\lambda_{\mathcal{H}}^{1}
$$

This proves that $u$ is a minimizer of (3.2). Clearly also $|u| \geq 0$ is a minimizer, so we may assume $u \geq 0$ a.e. Also, the Euler-Lagrange Eq. (1.8) is satisfied. Taking into account that $S(u) \geq 0$, we get

$$
\int_{\Omega}\left(p \frac{|\nabla u|^{p-2}}{\lambda^{p-2}}+q a(x) \frac{|\nabla u|^{q-2}}{\lambda^{q-2}}\right) \frac{\nabla u}{\lambda} \cdot \nabla \varphi \mathrm{d} x \geq 0 \text { for all } \varphi \in W_{0}^{1, \mathcal{H}}(\Omega), \varphi \geq 0,
$$


namely $u$ is a nonnegative supersolution for the equation

$$
-\operatorname{div}\left(p\left|\frac{\nabla u}{\lambda}\right|^{p-2} \frac{\nabla u}{\lambda}+q a(x)\left|\frac{\nabla u}{\lambda}\right|^{q-2} \frac{\nabla u}{\lambda}\right)=0 .
$$

Considering now radii $r_{2}>r_{1}>0, k \geq 0$, a cutoff $\eta$ with $\eta=1$ on $B_{r_{1}}$ and $\eta=0$ on $B_{r_{2}}^{c}$ and choosing the bounded test function $\varphi:=(u / \lambda-k)-\eta^{q}$, if $h(x, t):=t^{p-1}+a(x) t^{q-1}$, recalling that $p<q$ it holds

$$
q^{2} \int_{B_{r_{2}}} h\left(x, \frac{|\nabla u|}{\lambda}\right)\left(\frac{u}{\lambda}-k\right)_{-}|\nabla \eta| \eta^{q-1} \mathrm{~d} x \geq p \int_{B_{r_{2}}} \mathcal{H}\left(x, \frac{|\nabla u|}{\lambda}\right) \eta^{q} \chi_{\{u / \lambda \leq k\}} \mathrm{d} x .
$$

Young's inequality with exponents $\left(p^{\prime}, p\right)$ yields for some $c_{1}(p, q)>0$,

$$
\begin{aligned}
q^{2} \int_{B_{r_{2}}} & \left(\frac{|\nabla u|}{\lambda}\right)^{p-1}\left(\frac{u}{\lambda}-k\right)_{-}|\nabla \eta| \eta^{q-1} \mathrm{~d} x \\
& \leq \frac{p}{2} \int_{B_{r_{2}}}\left(\frac{|\nabla u|}{\lambda}\right)^{p} \eta^{p^{\prime}(q-1)} \chi_{\{u / \lambda \leq k\}} \mathrm{d} x+c_{1}(p, q) \int_{B_{r_{2}}}\left(\frac{u}{\lambda}-k\right)_{-}^{p}|\nabla \eta|^{p} \mathrm{~d} x \\
& \leq \frac{p}{2} \int_{B_{r_{2}}}\left(\frac{|\nabla u|}{\lambda}\right)^{p} \eta^{q} \chi_{\{u / \lambda \leq k\}} \mathrm{d} x+c_{1}(p, q) \int_{B_{r_{2}}}\left(\left(\frac{u}{\lambda}-k\right)_{-}\|\nabla \eta\|_{L^{\infty}}\right)^{p} \mathrm{~d} x,
\end{aligned}
$$

where we have used that $p^{\prime}(q-1) \geq q$, since $q>p$ by assumption. Analogously, using Young's inequality with exponents $\left(q^{\prime}, q\right)$ also yields, for some $c_{2}(p, q)>0$,

$$
\begin{aligned}
q^{2} \int_{B_{r_{2}}} a(x)\left(\frac{|\nabla u|}{\lambda}\right)^{q-1}\left(\frac{u}{\lambda}-k\right)_{-} \mid \nabla \eta \eta^{q-1} \mathrm{~d} x \\
\leq \frac{p}{2} \int_{B_{r_{2}}} a(x)\left(\frac{|\nabla u|}{\lambda}\right)^{q} \eta^{q} \chi_{\{u / \lambda \leq k\}} \mathrm{d} x \\
\quad+c_{2}(p, q) \int_{B_{r_{2}}} a(x)\left(\left(\frac{u}{\lambda}-k\right)_{-}\|\nabla \eta\|_{L^{\infty}}\right)^{q} \mathrm{~d} x .
\end{aligned}
$$

Whence, by absorbing the first two terms of the right-hand sides into (3.13), we conclude that

$$
\int_{B_{r_{1}}} \mathcal{H}\left(x, \frac{|\nabla u|}{\lambda}\right) \chi_{\{u / \lambda \leq k\}} \mathrm{d} x \leq c(p, q) \int_{B_{r_{2}}} \mathcal{H}\left(x,\left(\frac{u}{\lambda}-k\right)_{-} \frac{1}{\left(r_{2}-r_{1}\right)}\right) \mathrm{d} x
$$

for some positive constant $c(p, q)$, since $\|\nabla \eta\|_{L^{\infty}}$ is of order $\left(r_{2}-r_{1}\right)^{-1}$. It follows that the function $u / \lambda$ belongs to the De Giorgi class $D G_{-}$(cf. Section 6 of [5]). Then, in turn, by a slight modification of Theorem 3.5 of [5] (in order to allow supersolutions of Eq. (3.12)), $u$ satisfies the weak Harnack inequality, yielding

$$
\inf _{B_{r}(x)} u \geq \frac{1}{c}\left(f_{B_{2 r}(x)} u^{\varepsilon}(y) \mathrm{d} y\right)^{1 / \varepsilon},
$$

for some constants $c \geq 1$ and $\varepsilon \in(0,1)$ and for every ball $B_{2 r}(x) \subset \Omega$. This immediately yields $u>0$, by a standard argument. The boundedness of eigenfunctions follows by Sect. 3.2. Finally, the stability and symmetry properties follow by Theorems 3.5, 3.6 and 3.8. 
Remark 3.4 If $\Omega \subset \tilde{\Omega}$, then for all $u \in W_{0}^{1, \mathcal{H}}(\Omega)$, the extension by zero

$$
\tilde{u}:= \begin{cases}u & \text { in } \Omega, \\ 0 & \text { in } \tilde{\Omega} \backslash \Omega\end{cases}
$$

belongs to $W_{0}^{1, \tilde{\mathcal{H}}}(\tilde{\Omega})$, where $\tilde{\mathcal{H}}(x, t):=t^{p}+\tilde{a}(x) t^{q}$ for all $(x, t) \in \tilde{\Omega} \times[0, \infty)$, and $\tilde{a}$ extends by zero $a$ in $\tilde{\Omega} \backslash \Omega$. Indeed, being $W_{0}^{1, \mathcal{H}}(\Omega) \hookrightarrow W_{0}^{1, p}(\Omega)$, we can use a classical result in $W_{0}^{1, p}(\Omega)$ (see e.g., Proposition 9.18 of [11]) to obtain that

$$
\nabla \tilde{u}= \begin{cases}\nabla u & \text { in } \Omega, \\ 0 & \text { in } \tilde{\Omega} \backslash \Omega .\end{cases}
$$

Since $u \in W_{0}^{1, \mathcal{H}}(\Omega)$, there exists a sequence $\left(\varphi_{h}\right) \subset C_{0}^{\infty}(\Omega)$ such that $\left\|\nabla \varphi_{h}-\nabla u\right\|_{\mathcal{H}} \rightarrow 0$ as $h \rightarrow \infty$. By Proposition 2.1.11 of [23], norm convergence and modular convergence are equivalent, thus $\varrho_{\mathcal{H}}\left(\nabla \varphi_{h}-\nabla u\right) \rightarrow 0$ as $h \rightarrow \infty$. Extending by zero $a$ and each $\varphi_{h}$ in $\tilde{\Omega} \backslash \Omega$, we have $\left(\varphi_{h}\right) \subset C_{0}^{\infty}(\tilde{\Omega})$, and so

$$
\begin{aligned}
\varrho_{\mathcal{H}}\left(\nabla \varphi_{h}-\nabla u\right) & =\int_{\Omega}\left(\left|\nabla \varphi_{h}-\nabla u\right|^{p}+a(x)\left|\nabla \varphi_{h}-\nabla u\right|^{q}\right) \mathrm{d} x \\
& =\int_{\tilde{\Omega}}\left(\left|\nabla \varphi_{h}-\nabla \tilde{u}\right|^{p}+\tilde{a}(x)\left|\nabla \varphi_{h}-\nabla \tilde{u}\right|^{q}\right) \mathrm{d} x=\tilde{\varrho}_{\tilde{\mathcal{H}}}\left(\nabla \varphi_{h}-\nabla \tilde{u}\right) .
\end{aligned}
$$

Therefore, $\left\|\nabla \varphi_{h}-\nabla \tilde{u}\right\|_{L \tilde{\mathcal{H}}(\tilde{\Omega})} \rightarrow 0$ as $h \rightarrow \infty$ and so $\tilde{u} \in W_{0}^{1, \tilde{\mathcal{H}}}(\tilde{\Omega})$.

Theorem 3.5 (Stability in domains) Let $\left(\Omega_{h}\right)$ be a strictly increasing sequence of open subsets of $\mathbb{R}^{n}$ such that

$$
\Omega=\bigcup_{h=1}^{\infty} \Omega_{h}
$$

Then

$$
\lim _{h \rightarrow \infty} \lambda_{\mathcal{H}}^{1}\left(\Omega_{h}\right)=\lambda_{\mathcal{H}}^{1}
$$

(we omit the dependence when the domain is $\Omega$ ).

Proof Extending the functions $u \in W_{0}^{1, \mathcal{H}}\left(\Omega_{h}\right)$ as zero in $\Omega \backslash \Omega_{h}$, by Remark 3.4 we get $u \in W_{0}^{1, \mathcal{H}}(\Omega)$ and clearly

$$
\lambda_{\mathcal{H}}^{1}\left(\Omega_{1}\right) \geq \lambda_{\mathcal{H}}^{1}\left(\Omega_{2}\right) \geq \cdots \geq \lambda_{\mathcal{H}}^{1}
$$

On the other hand, by density, $\lambda_{\mathcal{H}}^{1}=\inf _{u \in C_{0}^{\infty}(\Omega) \backslash\{0\}}\|\nabla u\|_{\mathcal{H}} /\|u\|_{\mathcal{H}}$. So, fixed $\varepsilon>0$, we can find $\varphi \in C_{0}^{\infty}(\Omega)$ for which

$$
\lambda_{\mathcal{H}}^{1}>\frac{\|\nabla \varphi\|_{\mathcal{H}}}{\|\varphi\|_{\mathcal{H}}}-\varepsilon
$$

Since the support of $\varphi$ is compact, it is covered by a finite number of $\Omega_{h}$ 's, hence for $h$ sufficiently large $\operatorname{supp} \varphi \subset \Omega_{h}$. Whence,

$$
\lambda_{\mathcal{H}}^{1}\left(\Omega_{h}\right) \leq \frac{\|\nabla \varphi\|_{L^{\mathcal{H}}\left(\Omega_{h}\right)}}{\|\varphi\|_{L^{\mathcal{H}}\left(\Omega_{h}\right)}}=\frac{\|\nabla \varphi\|_{\mathcal{H}}}{\|\varphi\|_{\mathcal{H}}}
$$


and by (3.15)

$$
\lambda_{\mathcal{H}}^{1}>\lambda_{\mathcal{H}}^{1}\left(\Omega_{h}\right)-\varepsilon \text { for } h \text { large. }
$$

By the arbitrariness of $\varepsilon$

$$
\lambda_{\mathcal{H}}^{1} \geq \lim _{h \rightarrow \infty} \lambda_{\mathcal{H}}^{1}\left(\Omega_{h}\right)
$$

which, combined with (3.14), gives the conclusion.

Theorem 3.6 (Isoperimetric property) Let $a \equiv 1$ and $\Omega^{*}$ be the ball of $\mathbb{R}^{n}$ such that $\left|\Omega^{*}\right|=$ $|\Omega|$. Then, we have

$$
\lambda_{\mathcal{H}}^{1}\left(\Omega^{*}\right) \leq \lambda_{\mathcal{H}}^{1} .
$$

Moreover, if equality holds in (3.16), then $\Omega$ is a ball. In other words, balls uniquely minimize the first eigenvalue among sets with given $n$-dimensional Lebesgue measure.

Proof Let us prove (3.16). Let $u^{*}$ be the Schwarz symmetrization of a given nonnegative function $u \in W_{0}^{1, \mathcal{H}}(\Omega)$, namely the unique radially symmetric and decreasing function with

$$
\left|\left\{x \in \Omega^{*}: u^{*}(x)>t\right\}\right|=|\{x \in \Omega: u(x)>t\}| \text { for all } t>0 .
$$

Since $a \equiv 1$, by (i) of Proposition 2.15, we have

$$
W_{0}^{1, \mathcal{H}}(\Omega) \hookrightarrow W_{0}^{1, p}(\Omega), \quad W_{0}^{1, \mathcal{H}}(\Omega) \hookrightarrow W_{0}^{1, q}(\Omega) .
$$

Then, in light of the Pólya-Szegö's inequality, we get $u^{*} \in W_{0}^{1, p}\left(\Omega^{*}\right) \cap W_{0}^{1, q}\left(\Omega^{*}\right)$ and

$$
\varrho_{\mathcal{H}}^{*}\left(\nabla u^{*}\right)=\int_{\Omega^{*}}\left(\left|\nabla u^{*}\right|^{p}+\left|\nabla u^{*}\right|^{q}\right) \mathrm{d} x \leq \int_{\Omega}\left(|\nabla u|^{p}+|\nabla u|^{q}\right) \mathrm{d} x=\varrho_{\mathcal{H}}(\nabla u),
$$

so $u^{*} \in W_{0}^{1, \mathcal{H}}\left(\Omega^{*}\right)$. For $u \in W_{0}^{1, \mathcal{H}}(\Omega) \backslash\{0\}$,

$$
\begin{aligned}
\varrho_{\mathcal{H}}^{*}\left(\frac{\nabla u^{*}}{\|\nabla u\|_{\mathcal{H}}}\right) & =\frac{1}{\|\nabla u\|_{\mathcal{H}}^{p}} \int_{\Omega^{*}}\left|\nabla u^{*}\right|^{p} \mathrm{~d} x+\frac{1}{\|\nabla u\|_{\mathcal{H}}^{q}} \int_{\Omega^{*}}\left|\nabla u^{*}\right|^{q} \mathrm{~d} x \\
& \leq \frac{1}{\|\nabla u\|_{\mathcal{H}}^{p}} \int_{\Omega}|\nabla u|^{p} \mathrm{~d} x+\frac{1}{\|\nabla u\|_{\mathcal{H}}^{q}} \int_{\Omega}|\nabla u|^{q} \mathrm{~d} x=\varrho_{\mathcal{H}}\left(\frac{\nabla u}{\|\nabla u\|_{\mathcal{H}}}\right)=1
\end{aligned}
$$

which gives, by the unit ball property,

$$
\left\|\nabla u^{*}\right\|_{L^{\mathcal{H}}\left(\Omega^{*}\right)} \leq\|\nabla u\|_{\mathcal{H}} .
$$

On the other hand, since Schwarz symmetrization preserves all $L^{p}$-norms,

$$
\varrho_{\mathcal{H}}^{*}\left(u^{*}\right)=\int_{\Omega^{*}}\left(\left|u^{*}\right|^{p}+\left|u^{*}\right|^{q}\right) \mathrm{d} x=\int_{\Omega}\left(|u|^{p}+|u|^{q}\right) \mathrm{d} x=\varrho_{\mathcal{H}}(u) .
$$

Thus, again the unit ball property gives

$$
\left\|u^{*}\right\|_{L^{\mathcal{H}}\left(\Omega^{*}\right)}=\|u\|_{\mathcal{H}} .
$$

Hence, if we take $u=u_{\mathcal{H}}^{1} \geq 0$ a.e., we obtain

$$
\lambda_{\mathcal{H}}^{1}\left(\Omega^{*}\right) \leq \frac{\left\|\nabla\left(u_{\mathcal{H}}^{1}\right)^{*}\right\|_{L^{\mathcal{H}}\left(\Omega^{*}\right)}}{\left\|\left(u_{\mathcal{H}}^{1}\right)^{*}\right\|_{L^{\mathcal{H}}\left(\Omega^{*}\right)}} \leq \frac{\left\|\nabla u_{\mathcal{H}}^{1}\right\|_{\mathcal{H}}}{\left\|u_{\mathcal{H}}^{1}\right\|_{\mathcal{H}}}=\lambda_{\mathcal{H}}^{1},
$$


which concludes the proof. Assume now that equality holds in inequality (3.16) and consider a first nonnegative eigenfunction $w$ for $\lambda_{\mathcal{H}}^{1}$. Then, recalling that $\left\|w^{*}\right\|_{L^{\mathcal{H}}\left(\Omega^{*}\right)}=\|w\|_{\mathcal{H}}$, we conclude by the very definition of $\lambda_{\mathcal{H}}^{1}$ that $\left\|\nabla w^{*}\right\|_{L^{\mathcal{H}}\left(\Omega^{*}\right)}=\|\nabla w\|_{\mathcal{H}}$. This, in light of (2.1) gives

$$
\varrho_{\mathcal{H}}^{*}\left(\frac{\nabla w^{*}}{\|\nabla w\|_{\mathcal{H}}}\right)=\varrho_{\mathcal{H}}\left(\frac{\nabla w}{\|\nabla w\|_{\mathcal{H}}}\right) .
$$

Since, separately, we have

$$
\int_{\Omega^{*}}\left|\nabla w^{*}\right|^{p} \mathrm{~d} x \leq \int_{\Omega}|\nabla w|^{p} \mathrm{~d} x, \quad \int_{\Omega^{*}}\left|\nabla w^{*}\right|^{q} \mathrm{~d} x \leq \int_{\Omega}|\nabla w|^{q} \mathrm{~d} x,
$$

we deduce from identity (3.17) (in which the denominators agree) that

$$
\left\|\nabla w^{*}\right\|_{L^{p}\left(\Omega^{*}\right)}=\|\nabla w\|_{L^{p}(\Omega)} .
$$

This implies (see e.g., [30]) that the superlevels of $w$ are balls and, thus, $\Omega$ is a ball, completing the proof.

Remark 3.7 Theorem 3.6 represents an extension to the double phase case of the so-called Faber-Krahn inequality (cf. [30] for the single phase case). It was firstly shown by Faber and Krahn $[24,35,36]$ that the first eigenvalue of $-\Delta$ on a bounded open set of $\mathbb{R}^{2}$ of given area attains its minimum value if and only if is a disk, namely the gravest principal tone is obtained in the case of a circular membrane, as conjectured by Lord Rayleigh in 1877 [55].

A subset $H$ of $\mathbb{R}^{n}$ is called a polarizer if it is a closed affine half-space of $\mathbb{R}^{n}$, namely the set of points $x$ which satisfy $\alpha \cdot x \leq \beta$ for some $\alpha \in \mathbb{R}^{n}$ and $\beta \in \mathbb{R}$ with $|\alpha|=1$. Given $x$ in $\mathbb{R}^{n}$ and a polarizer $H$ the reflection of $x$ with respect to the boundary of $H$ is denoted by $x_{H}$. The polarization of a function $u: \mathbb{R}^{n} \rightarrow \mathbb{R}^{+}$by a polarizer $H$ is the function $u^{H}: \mathbb{R}^{n} \rightarrow \mathbb{R}^{+}$ defined by

$$
u^{H}(x)= \begin{cases}\max \left\{u(x), u\left(x_{H}\right)\right\}, & \text { if } x \in H \\ \min \left\{u(x), u\left(x_{H}\right)\right\}, & \text { if } x \in \mathbb{R}^{n} \backslash H .\end{cases}
$$

The polarization $C^{H} \subset \mathbb{R}^{n}$ of a set $C \subset \mathbb{R}^{n}$ is defined as the unique set which satisfies $\chi_{C^{H}}=\left(\chi_{C}\right)^{H}$, where $\chi$ denotes the characteristic function. This operation should not be confused with $C_{H}$ which denotes the reflection of $C$ with respect to $\partial H$. The polarization $u^{H}$ of a positive function $u$ defined on $C \subset \mathbb{R}^{n}$ is the restriction to $C^{H}$ of the polarization of the extension $\tilde{u}: \mathbb{R}^{n} \rightarrow \mathbb{R}^{+}$of $u$ by zero outside $C$. The polarization of a function which may change sign is defined by $u^{H}:=|u|^{H}$, for any given polarizer $H$.

Theorem 3.8 (Partial symmetries) Let $a \equiv 1, H \subset \mathbb{R}^{n}$ be a half-space and assume that $\Omega=\Omega^{H}$. Then, there exists a nonnegative first eigenfunction $u \in W_{0}^{1, \mathcal{H}}(\Omega)$ such that $u=u^{H}$.

Proof Let $u \in W_{0}^{1, \mathcal{H}}(\Omega)$ with $u \geq 0$ a.e. be given. Then, since $u \in W_{0}^{1, p}(\Omega)$ and $u \in$ $W_{0}^{1, q}(\Omega)$, by Proposition 2.3 of [57], we have

$$
\varrho \mathcal{H}\left(\nabla u^{H}\right)=\int_{\Omega}\left(\left|\nabla u^{H}\right|^{p}+\left|\nabla u^{H}\right|^{q}\right) \mathrm{d} x=\int_{\Omega}\left(|\nabla u|^{p}+|\nabla u|^{q}\right) \mathrm{d} x=\varrho \mathcal{H}(\nabla u),
$$

so $u^{H} \in W_{0}^{1, \mathcal{H}}(\Omega)$ and by the unit ball property

$$
\left\|\nabla u^{H}\right\|_{\mathcal{H}}=\|\nabla u\|_{\mathcal{H}} .
$$


Analogously, we have $\left\|u^{H}\right\|_{\mathcal{H}}=\|u\|_{\mathcal{H}}$. We want to apply the symmetric Ekeland variational principle with constraint (see Section 2.4, p. 334 of [54], see also [53]) by choosing

$$
X:=W_{0}^{1, \mathcal{H}}(\Omega), \quad S:=W_{0}^{1, \mathcal{H}}\left(\Omega, \mathbb{R}^{+}\right), \quad V:=L^{p}(\Omega), \quad f(u):=\|\nabla u\|_{\mathcal{H}}, \quad u \in X .
$$

Let $\left(v_{h}\right) \subset \mathcal{M}$ be a nonnegative minimization sequence, namely

$$
\lim _{h \rightarrow \infty}\left\|\nabla v_{h}\right\|_{\mathcal{H}}=\lambda_{\mathcal{H}}^{1}
$$

Then, there exists a new minimization sequence $\left(\tilde{v}_{h}\right) \subset \mathcal{M}$ such that

$$
\left\|\left|\tilde{v}_{h}\right|^{H}-\tilde{v}_{h}\right\|_{p} \rightarrow 0, \quad \text { as } h \rightarrow \infty .
$$

Up to a subsequence $\left(\tilde{v}_{h_{j}}\right)$ converges weakly to $u$ in $W_{0}^{1, \mathcal{H}}(\Omega)$ and, in light of Proposition 2.18-(iii), we obtain $\left\|\tilde{v}_{h_{j}}-u\right\|_{\mathcal{H}} \rightarrow 0$ as $j \rightarrow \infty$ (and hence $\left\|\tilde{v}_{h_{j}}-u\right\|_{p} \rightarrow 0$ as $j \rightarrow \infty)$ so that $\|u\|_{\mathcal{H}}=1$. This easily implies that $u$ is a minimizer of (3.1). Finally, observing that (standard contractivity of the polarization in the $L^{p}$-norm)

$$
\left\|\left|\tilde{v}_{h_{j}}\right|^{H}-|u|^{H}\right\|_{p} \leq\left\|\left|\tilde{v}_{h_{j}}\right|-|u|\right\|_{p} \leq\left\|\tilde{v}_{h_{j}}-u\right\|_{p} \rightarrow 0, \quad \text { as } h \rightarrow \infty,
$$

which, taking into account (3.19), yields

$\left\||u|^{H}-u\right\|_{p} \leq\left\|\left|\tilde{v}_{h_{j}}\right|^{H}-|u|^{H}\right\|_{p}+\left\|\left|\tilde{v}_{h_{j}}\right|^{H}-\tilde{v}_{h_{j}}\right\|_{p}+\left\|\tilde{v}_{h_{j}}-u\right\|_{p} \rightarrow 0, \quad$ as $h \rightarrow \infty$,

which yields $|u|^{H}=u$. Hence $u \geq 0$ and $u^{H}=u$, concluding the proof.

\subsection{Large exponents}

The next result concerns the behavior of the first eigenvalue $\lambda_{\mathcal{H}}^{1}$, when the exponents $p$ and $q$ of the $N$-function $\mathcal{H}$ are replaced by $h p$ and $h q$, respectively, and $h$ goes to infinity. The passage to infinity was first studied in [34] for the $p$-Laplacian operator and then in [28] for the $p(x)$-Laplacian.

Clearly, in order to study the $\infty$-eigenvalue problem, we do not require any bound from above on the exponents $h q$, we only assume that $1<p<q$. Furthermore, throughout this subsection, we use the rescaled modular

$$
\tilde{\varrho}_{\mathcal{H}}(u):=\frac{1}{|\Omega|+\|a\|_{1}} \int_{\Omega}\left(|u|^{p}+a(x)|u|^{q}\right) \mathrm{d} x
$$

and we denote by $\left\||\cdot \||_{\mathcal{H}}\right.$ the corresponding norm. It is easy to see that $\||\cdot \||_{\mathcal{H}}$ is equivalent to $\|\cdot\|_{\mathcal{H}}$, more precisely, by (2.1.5) of [23] and by the unit ball property,

$$
\begin{array}{ll}
\left\|\left|u\left\|\left.\right|_{\mathcal{H}} \leq\right\| u\left\|_{\mathcal{H}} \leq\left(|\Omega|+\|a\|_{1}\right)\right\|\right| u\right\|_{\mathcal{H}}, & \text { if }|\Omega|+\|a\|_{1} \geq 1, \\
\left(|\Omega|+\|a\|_{1}\right)\left\|\left|u\left\|_{\mathcal{H}} \leq\right\| u\left\|_{\mathcal{H}} \leq\right\|\right| u\right\|_{\mathcal{H}}, & \text { if }|\Omega|+\|a\|_{1}<1 .
\end{array}
$$

We introduce the distance function

$$
\delta(x):=\operatorname{dist}(x, \partial \Omega) \text { for all } x \in \Omega .
$$

We recall that $\delta$ is Lipschitz continuous and that $\nabla \delta=1$ a.e. in $\Omega$. We define

$$
\lambda_{\infty}^{1}:=\inf _{u \in W_{0}^{1, \infty}(\Omega) \backslash\{0\}} \frac{\|\nabla u\|_{\infty}}{\|u\|_{\infty}}
$$


Proceeding as in Section 4 of [28], it is easy to see that the minimum in (3.21) is reached on the distance function and so

$$
\lambda_{\infty}^{1}=\frac{1}{\|\delta\|_{\infty}}=\frac{1}{R}
$$

where $R$ is the so-called inradius, i.e., the radius of the largest ball inscribed in $\Omega$.

For all $h \in \mathbb{N}$, put

$$
(h \mathcal{H})(x, t):=t^{h p}+a(x) t^{h q} \text { for all }(x, t) \in \Omega \times[0, \infty) .
$$

Lemma 3.9 Let $u \in L^{\infty}(\Omega)$ then

$$
\lim _{h \rightarrow \infty}\left\|\left|u\left\|\left.\right|_{h \mathcal{H}}=\right\| u \|_{\infty} .\right.\right.
$$

Proof First, we want to show that

$$
\limsup _{h \rightarrow \infty}\left\|\left|u\left\|\left.\right|_{h \mathcal{H}} \leq\right\| u \|_{\infty} .\right.\right.
$$

To this aim, it is enough to consider only those indices $h$ for which $\left\|\left|u\left\|\left.\right|_{h \mathcal{H}}>\right\| u \|_{\infty}\right.\right.$,

$$
\begin{aligned}
1 & =\left[\tilde{\varrho}_{h \mathcal{H}}\left(\frac{u}{\left\||u \||_{h \mathcal{H}}\right.}\right)\right]^{\frac{1}{h p}}=\left[\int_{\Omega}\left(\left|\frac{u}{\|\mid u\|_{h \mathcal{H}}}\right|^{h p}+a(x)\left|\frac{u}{\left\||u \||_{h \mathcal{H}}\right.}\right|^{h q}\right) \frac{1}{|\Omega|+\|a\|_{1}} \mathrm{~d} x\right]^{\frac{1}{h p}} \\
& \leq\left[\int_{\Omega}\left(\frac{\|u\|_{\infty}}{\|\mid u\|_{h \mathcal{H}}}\right)^{h p} \frac{1+a(x)}{|\Omega|+\|a\|_{1}} \mathrm{~d} x\right]^{\frac{1}{h p}}=\frac{\|u\|_{\infty}}{\|\mid u\|_{h \mathcal{H}}} .
\end{aligned}
$$

This implies (3.22). Now, in order to prove

$$
\liminf _{h \rightarrow \infty}\left\|\left|u\left\|\left.\right|_{h \mathcal{H}} \geq\right\| u \|_{\infty},\right.\right.
$$

we assume that $\|u\|_{\infty}>0$ (the other case is obvious). Then, given $\varepsilon>0$, we can find a set $A_{\varepsilon} \subset \Omega$, with $\left|A_{\varepsilon}\right|>0$, such that $|u(x)|>\|u\|_{\infty}-\varepsilon$ for all $x \in A_{\varepsilon}$. We consider only those indices $h$ for which $\|\mid u\|_{h \mathcal{H}} \geq\|u\|_{\infty}-\varepsilon$ and we have

$$
\begin{aligned}
1 & =\left[\tilde{\varrho}_{h \mathcal{H}}\left(\frac{u}{\|\mid u\|_{h \mathcal{H}}}\right)\right]^{\frac{1}{h p}} \geq\left[\int_{A_{\varepsilon}}\left(\left|\frac{u}{\|\mid u\|_{h \mathcal{H}}}\right|^{h p}+a(x)\left|\frac{u}{\|\mid u\|_{h \mathcal{H}}}\right|^{h q}\right) \frac{1}{|\Omega|+\|a\|_{1}} \mathrm{~d} x\right]^{\frac{1}{h p}} \\
& >\left[\int_{A_{\varepsilon}}\left(\frac{\|u\|_{\infty}-\varepsilon}{\|\mid u\|_{h \mathcal{H}}}\right)^{h p} \frac{1}{|\Omega|+\|a\|_{1}} \mathrm{~d} x\right]^{\frac{1}{h p}}=\left(\frac{\left|A_{\varepsilon}\right|}{|\Omega|+\|a\|_{1}}\right)^{\frac{1}{h p}} \frac{\|u\|_{\infty}-\varepsilon}{\|\mid u\| \|_{h \mathcal{H}}},
\end{aligned}
$$

which gives $\lim \inf _{h \rightarrow \infty}\left\|\left|u\left\|\left.\right|_{h \mathcal{H}} \geq\right\| u \|_{\infty}-\varepsilon\right.\right.$ and by the arbitrariness of $\varepsilon$ we conclude.

We remark that the same property stated in Lemma 3.9 holds if we endow the space $L^{\mathcal{H}}(\Omega)$ with the standard modular $\varrho_{\mathcal{H}}$ and the corresponding norm $\|\cdot\|_{\mathcal{H}}$. Furthermore, if we consider the norm in $L^{r}(\Omega)$

$$
\|\mid u\| \|_{r}:=\frac{\|u\|_{r}}{\left(|\Omega|+\|a\|_{1}\right)^{1 / r}},
$$

the classical result $\lim _{r \rightarrow \infty}\left\|\left|u\left\|\left.\right|_{r}=\right\| u \|_{\infty}\right.\right.$ continues to hold. 
Theorem 3.10 There holds

$$
\lim _{h \rightarrow \infty} \tilde{\lambda}_{h \mathcal{H}}^{1}=\lambda_{\infty}^{1}
$$

where

$$
\tilde{\lambda}_{h \mathcal{H}}^{1}:=\inf _{u \in W_{0}^{1, \mathcal{H}}(\Omega) \backslash\{0\}} \frac{\|\mid \nabla u\|_{\mathcal{H}}}{\left\||u \||_{\mathcal{H}}\right.} .
$$

Proof By the definition of $\tilde{\lambda}_{h \mathcal{H}}^{1}$, using $\delta$ as test function, we have

$$
\tilde{\lambda}_{h \mathcal{H}}^{1} \leq \frac{\left\||\nabla \delta \||_{h \mathcal{H}}\right.}{\left\||\delta \||_{h \mathcal{H}}\right.} \text { for all } h \in \mathbb{N} .
$$

Thus, passing to the limit superior and taking into account Lemma 3.9, we obtain

$$
\limsup _{h \rightarrow \infty} \tilde{\lambda}_{h \mathcal{H}}^{1} \leq \frac{\|\nabla \delta\|_{\infty}}{\|\delta\|_{\infty}}=\lambda_{\infty}^{1} .
$$

Let $\left(u_{h}\right)$ be the sequence of first eigenfunctions corresponding to $\tilde{\lambda}_{h \mathcal{H}}^{1}$, with $\left\|\left|u_{h} \|\right|_{h \mathcal{H}}=1\right.$ for all $h$. Pick any subsequence $\left(u_{h_{j}}\right)$ of $\left(u_{h}\right)$. Then, $\tilde{\lambda}_{h_{j} \mathcal{H}}^{1}=\left\|\left|\nabla u_{h_{j}} \|\right|_{h_{j} \mathcal{H}}\right.$ and, by (3.23), the sequence $\left(\left\|\left|\nabla u_{h_{j}} \|\right|_{h \mathcal{H}}\right)\right.$ is bounded. Therefore, in correspondence to any $r \in[1, \infty)$ there is an integer $j_{r}$ such that $h_{j} p \geq r$ for all $j \geq j_{r}$, and consequently

$$
W_{0}^{1, h_{j} \mathcal{H}}(\Omega) \hookrightarrow W_{0}^{1, r}(\Omega) \hookrightarrow \hookrightarrow L^{r}(\Omega) \quad \text { for all } j \geq j_{r} .
$$

Hence, $\left(u_{h_{j}}\right)$ is definitely bounded in the reflexive Banach space $W_{0}^{1, r}(\Omega)$ and we can extract a subsequence, still denoted by $\left(u_{h_{j}}\right)$, for which

$$
\nabla u_{h_{j}} \rightarrow \nabla u_{\infty} \text { and } u_{h_{j}} \rightarrow u_{\infty} \text { in } L^{r}(\Omega) .
$$

By the arbitrariness of $r$ and the fact that $\Omega$ is bounded, we get $u_{\infty} \in W_{0}^{1, \infty}(\Omega)$. In particular, $u_{h_{j}} \rightarrow u_{\infty}$ also in $L^{\infty}(\Omega)$, since, by Proposition 2.15-(ii), $u_{h_{j}} \in L^{\infty}(\Omega)$ for $j$ large. By Hölder's inequality, for all $u \in L^{r}(\Omega)$

$$
\begin{aligned}
\tilde{\varrho}_{r}(u) & =\int_{\Omega} \frac{|u|^{r}}{|\Omega|+\|a\|_{1}} \mathrm{~d} x \leq\left(\int_{\Omega} \frac{|u|^{h_{j} p}}{|\Omega|+\|a\|_{1}} \mathrm{~d} x\right)^{\frac{r}{h_{j} p}}\left(\int_{\Omega} \frac{1+a(x)}{|\Omega|+\|a\|_{1}} \mathrm{~d} x\right)^{\frac{h_{j} p-r}{h_{j} p}} \\
& \leq\left(\tilde{\varrho}_{h_{j}} \mathcal{H}(u)\right)^{\frac{r}{h_{j} p}},
\end{aligned}
$$

whence

$$
\left.\left\|\left|u\left\|\left.\right|_{r} \leq\right\|\right| u\right\|\right|_{h_{j} \mathcal{H}} \text { for all } j \geq j_{r},
$$

by the unit ball property. We know that $u_{h_{j}} \in L^{\infty}(\Omega)$ for $j$ large, so

$$
\tilde{\varrho}_{h_{j} \mathcal{H}}\left(\frac{u_{h_{j}}}{\left\|u_{h_{j}}\right\|_{\infty}}\right) \leq \int_{\Omega}\left(\left\|\frac{u_{h_{j}}}{\left\|u_{h_{j}}\right\|_{\infty}}\right\|_{\infty}^{h_{j} p}+a(x)\left\|\frac{u_{h_{j}}}{\left\|u_{h_{j}}\right\|_{\infty}}\right\|_{\infty}^{h_{j} q}\right) \frac{1}{|\Omega|+\|a\|_{1}} \mathrm{~d} x=1 .
$$

By the unit ball property, we obtain

$$
1=\left\|\left|u_{h_{j}}\left\|\left.\right|_{h_{j}} \mathcal{H} \leq\right\| u_{h_{j}} \|_{\infty} .\right.\right.
$$


By the weak lower semicontinuity of the $W_{0}^{1, r}(\Omega)$-norm and by virtue of (3.24) and (3.25),

$$
\begin{aligned}
\frac{\left\|\left|\nabla u_{\infty} \|\right|_{r}\right.}{\left\|\left|u_{\infty} \|\right|_{r}\right.} & \leq \liminf _{j \rightarrow \infty} \frac{\left\|\left|\nabla u_{h_{j}} \|\right|_{r}\right.}{\left\|\left|u_{h_{j}} \|\right|_{r}\right.} \leq \liminf _{j \rightarrow \infty} \frac{\left\|\left|\nabla u_{h_{j}} \|\right|_{h_{j}} \mathcal{H}\right.}{\left\|\left|u_{h_{j}} \|\right|_{r}\right.} \\
& \leq \liminf _{j \rightarrow \infty}\left(\left\|\left|\nabla u_{h_{j}} \|\right|_{h_{j}} \mathcal{H} \frac{\left\|u_{h_{j}}\right\|_{\infty}}{\left\|\left|u_{h_{j}} \|\right|_{r}\right.}\right)=\frac{\left\|u_{\infty}\right\|_{\infty}}{\left\|\left|u_{\infty} \|\right|_{r}\right.} \liminf _{j \rightarrow \infty} \tilde{\lambda}_{h_{j} \mathcal{H}}^{1}\right.
\end{aligned}
$$

Hence,

$$
\lambda_{\infty}^{1} \leq \frac{\left\|\nabla u_{\infty}\right\|_{\infty}}{\left\|u_{\infty}\right\|_{\infty}}=\lim _{r \rightarrow \infty} \frac{\left.\left\|\nabla u_{\infty}\right\|\right|_{r}}{\left\|\left|u_{\infty} \|\right|_{r}\right.} \leq \lim _{r \rightarrow \infty} \frac{\left\|u_{\infty}\right\|_{\infty}}{\left\|\mid u_{\infty}\right\| \|_{r}} \liminf _{j \rightarrow \infty} \tilde{\lambda}_{h_{j} \mathcal{H}}^{1}=\liminf _{j \rightarrow \infty} \tilde{\lambda}_{h_{j} \mathcal{H}}^{1},
$$

which, combined with (3.23), gives

$$
\lambda_{\infty}^{1}=\lim _{j \rightarrow \infty} \tilde{\lambda}_{h_{j} \mathcal{H}}^{1}
$$

Finally, we conclude the proof by the arbitrariness of the subsequence.

Remark 3.11 By (3.20), in terms of the first eigenvalues $\lambda_{h \mathcal{H}}^{1}$, Theorem 3.10 gives

$$
\begin{aligned}
& \frac{1}{|\Omega|+\|a\|_{1}} \lambda_{\infty}^{1} \leq \liminf _{h \rightarrow \infty} \lambda_{h \mathcal{H}}^{1} \leq \limsup _{h \rightarrow \infty} \lambda_{h \mathcal{H}}^{1} \leq\left(|\Omega|+\|a\|_{1}\right) \lambda_{\infty}^{1}, \quad \text { if }|\Omega|+\|a\|_{1} \geq 1, \\
& \left(|\Omega|+\|a\|_{1}\right) \lambda_{\infty}^{1} \leq \liminf _{h \rightarrow \infty} \lambda_{h \mathcal{H}}^{1} \leq \limsup _{h \rightarrow \infty} \lambda_{h \mathcal{H}}^{1} \leq \frac{1}{|\Omega|+\|a\|_{1}} \lambda_{\infty}^{1}, \quad \text { if }|\Omega|+\|a\|_{1}<1 .
\end{aligned}
$$

\subsection{Closedness of the spectrum}

Theorem 3.12 (Closedness of $\Lambda$ ) Assume that (1.7) holds, then the spectrum is a closed set.

Proof Let $\left(\lambda_{h}\right) \subset \Lambda$ be a sequence of eigenvalues of (3.3) converging to a certain $\lambda<\infty$. Let us denote by $\left(u_{h}\right)$ the sequence of the corresponding eigenfunctions such that $\left\|u_{h}\right\|_{\mathcal{H}}=1$ for all $h$. Then, we have

$$
\begin{aligned}
\int_{\Omega} & {\left[p\left(\frac{\left|\nabla u_{h}\right|}{\lambda_{h}}\right)^{p-2}+q a(x)\left(\frac{\left|\nabla u_{h}\right|}{\lambda_{h}}\right)^{q-2}\right] \frac{\nabla u_{h}}{\lambda_{h}} \cdot \nabla v \mathrm{~d} x } \\
& =\lambda_{h} S\left(u_{h}\right) \int_{\Omega}\left(p\left|u_{h}\right|^{p-2}+q a(x)\left|u_{h}\right|^{q-2}\right) u_{h} v \mathrm{~d} x \quad \text { for all } v \in W_{0}^{1, \mathcal{H}}(\Omega) \text { and } h \in \mathbb{N},
\end{aligned}
$$

and, by normalization, $\lambda_{h}=\left\|\nabla u_{h}\right\|_{\mathcal{H}}$. Therefore, $\left(u_{h}\right)$ is bounded in the reflexive Banach space $W_{0}^{1, \mathcal{H}}(\Omega)$ and it admits a subsequence $\left(u_{h_{j}}\right)$ such that $u_{h_{j}} \rightarrow u$ in $W_{0}^{1, \mathcal{H}}(\Omega)$ as $j \rightarrow \infty$. Thus, by Proposition 2.18-(iii), $u_{h_{j}} \rightarrow u$ in $L^{\mathcal{H}}(\Omega)$ strongly as $j \rightarrow \infty$, yielding $\|u\|_{\mathcal{H}}=1$ (which, in particular, provides $u \neq 0$ ). We claim that $u$ is an eigenfunction with corresponding eigenvalue $\lambda$, i.e., that the following distributional identity is satisfied for all $v \in C_{0}^{\infty}(\Omega)$,

$$
\begin{aligned}
& \int_{\Omega}\left[p\left(\frac{|\nabla u|}{\lambda}\right)^{p-2}+q a(x)\left(\frac{|\nabla u|}{\lambda}\right)^{q-2}\right] \frac{\nabla u}{\lambda} \cdot \nabla v \mathrm{~d} x \\
& =\lambda S(u) \int_{\Omega}\left(p|u|^{p-2}+q a(x)|u|^{q-2}\right) u v \mathrm{~d} x .
\end{aligned}
$$


In order to prove that, we shall pass to the limit in (3.26). First, being $L^{\mathcal{H}}(\Omega) \hookrightarrow L^{p}(\Omega)$, by the dominated convergence theorem,

$$
\int_{\Omega}\left|u_{h_{j}}\right|^{p-2} u_{h_{j}} v \mathrm{~d} x \rightarrow \int_{\Omega}|u|^{p-2} u v \mathrm{~d} x \text { for all } v \in C_{0}^{\infty}(\Omega)
$$

up to a subsequence. Moreover, being $L^{\mathcal{H}}(\Omega) \hookrightarrow L_{a}^{q}(\Omega), a^{1 / q} u_{h_{j}} \rightarrow a^{1 / q} u$ in $L^{q}(\Omega)$ and so, up to a subsequence, there exists $\omega \in L^{q}(\Omega)$ such that $\left|a^{1 / q} u_{h_{j}}\right| \leq \omega$ for all $j$. Therefore,

$$
a\left|u_{h_{j}}\right|^{q-1}|v|=a^{(q-1) / q}\left|u_{h_{j}}\right|^{q-1} a^{1 / q}|v| \leq \omega^{q-1} a^{1 / q}|v| \in L^{1}(\Omega) \text { for all } j,
$$

and so, the dominated convergence theorem implies

$$
\int_{\Omega} a(x)\left|u_{h_{j}}\right|^{q-2} u_{h_{j}} v \mathrm{~d} x \rightarrow \int_{\Omega} a(x)|u|^{q-2} u v \mathrm{~d} x \text { for all } v \in C_{0}^{\infty}(\Omega) .
$$

Therefore, by (3.27)-(3.28), there exists a subsequence, still denoted by $\left(u_{h_{j}}\right)$, for which the following limit holds for all $v \in C_{0}^{\infty}(\Omega)$,

$$
\lim _{j \rightarrow \infty} \int_{\Omega}\left(p\left|u_{h_{j}}\right|^{p-2}+q a(x)\left|u_{h_{j}}\right|^{q-2}\right) u_{h_{j}} v \mathrm{~d} x=\int_{\Omega}\left(p|u|^{p-2}+q a(x)|u|^{q-2}\right) u v \mathrm{~d} x .
$$

Now, by (3.26) with $v=\frac{u_{h_{j}}}{\lambda_{h_{j}}}-\frac{u}{\lambda}$, we get

$$
\begin{aligned}
\int_{\Omega} & {\left[\left(p\left|\frac{\nabla u_{h_{j}}}{\lambda_{h_{j}}}\right|^{p-2}+q a(x)\left|\frac{\nabla u_{h_{j}}}{\lambda_{h_{j}}}\right|^{q-2}\right) \frac{\nabla u_{h_{j}}}{\lambda_{h_{j}}}\right.} \\
& \left.-\left(p\left|\frac{\nabla u}{\lambda}\right|^{p-2}+q a(x)\left|\frac{\nabla u}{\lambda}\right|^{q-2}\right) \frac{\nabla u}{\lambda}\right] \cdot \nabla\left(\frac{u_{h_{j}}}{\lambda_{h_{j}}}-\frac{u}{\lambda}\right) \mathrm{d} x \\
= & \lambda_{h_{j}} S\left(u_{h_{j}}\right) \int_{\Omega}\left(p\left|u_{h_{j}}\right|^{p-2}+q a(x)\left|u_{h_{j}}\right|^{q-2}\right) u_{h_{j}}\left(\frac{u_{h_{j}}}{\lambda_{h_{j}}}-\frac{u}{\lambda}\right) \mathrm{d} x \\
& -\int_{\Omega}\left(p\left|\frac{\nabla u}{\lambda}\right|^{p-2}+q a(x)\left|\frac{\nabla u}{\lambda}\right|^{q-2}\right) \frac{\nabla u}{\lambda} \cdot \nabla\left(\frac{u_{h_{j}}}{\lambda_{h_{j}}}-\frac{u}{\lambda}\right) \mathrm{d} x .
\end{aligned}
$$

Passing to the limit under integral sign (we can reason as for (3.27) and (3.28)), and observing that $S\left(u_{h_{j}}\right) \leq q$ for all $j$,

$$
\lim _{j \rightarrow \infty} \lambda_{h_{j}} S\left(u_{h_{j}}\right) \int_{\Omega}\left(p\left|u_{h_{j}}\right|^{p-2}+q a(x)\left|u_{h_{j}}\right|^{q-2}\right) u_{h_{j}}\left(\frac{u_{h_{j}}}{\lambda_{h_{j}}}-\frac{u}{\lambda}\right) \mathrm{d} x=0 .
$$

Furthermore, since $\frac{\nabla u_{h_{j}}}{\lambda_{h_{j}}} \rightarrow \frac{\nabla u}{\lambda}$ in $\left[L^{\mathcal{H}}(\Omega)\right]^{n}$ and being $L^{\mathcal{H}}(\Omega) \hookrightarrow L^{p}(\Omega)$, we have

$$
\lim _{j \rightarrow \infty} \int_{\Omega}\left|\frac{\nabla u}{\lambda}\right|^{p-2} \frac{\nabla u}{\lambda} \cdot \nabla\left(\frac{u_{h_{j}}}{\lambda_{h_{j}}}-\frac{\nabla u}{\lambda}\right) \mathrm{d} x=0 .
$$

Analogously, $L^{\mathcal{H}}(\Omega) \hookrightarrow L_{a}^{q}(\Omega)$ and for the dual spaces the reverse embedding holds. It is easy to see that the functional 


$$
F: f \in\left[L_{a}^{q}(\Omega)\right]^{n} \mapsto \int_{\Omega} a(x)\left|\frac{\nabla u}{\lambda}\right|^{q-2} \frac{\nabla u}{\lambda} \cdot f \mathrm{~d} x
$$

belongs to $\left(\left[L_{a}^{q}(\Omega)\right]^{n}\right)^{\prime} \subset\left(\left[L^{\mathcal{H}}(\Omega)\right]^{n}\right)^{\prime}$. Since in particular $\frac{\nabla u_{h_{j}}}{\lambda_{h_{j}}} \rightarrow \frac{\nabla u}{\lambda}$ in $\left[L_{a}^{q}(\Omega)\right]^{n}$, we get

$$
\lim _{j \rightarrow \infty} \int_{\Omega} a(x)\left|\frac{\nabla u}{\lambda}\right|^{q-2} \frac{\nabla u}{\lambda} \cdot \nabla\left(\frac{u_{h_{j}}}{\lambda_{h_{j}}}-\frac{\nabla u}{\lambda}\right) \mathrm{d} x=0 .
$$

Then, by (3.30),

$$
\begin{aligned}
\lim _{j \rightarrow \infty} \int_{\Omega} & {\left[\left(p\left|\frac{\nabla u_{h_{j}}}{\lambda_{h_{j}}}\right|^{p-2}+q a(x)\left|\frac{\nabla u_{h_{j}}}{\lambda_{h_{j}}}\right|^{q-2}\right) \frac{\nabla u_{h_{j}}}{\lambda_{h_{j}}}\right.} \\
& \left.-\left(p\left|\frac{\nabla u}{\lambda}\right|^{p-2}+q a(x)\left|\frac{\nabla u}{\lambda}\right|^{q-2}\right) \frac{\nabla u}{\lambda}\right] \cdot \nabla\left(\frac{u_{h_{j}}}{\lambda_{h_{j}}}-\frac{u}{\lambda}\right) \mathrm{d} x=0,
\end{aligned}
$$

and consequently

$$
\begin{aligned}
& \lim _{j \rightarrow \infty} \int_{\Omega} p\left(\left|\frac{\nabla u_{h_{j}}}{\lambda_{h_{j}}}\right|^{p-2} \frac{\nabla u_{h_{j}}}{\lambda_{h_{j}}}-\left|\frac{\nabla u}{\lambda}\right|^{p-2} \frac{\nabla u}{\lambda}\right) \cdot \nabla\left(\frac{u_{h_{j}}}{\lambda_{h_{j}}}-\frac{u}{\lambda}\right) \mathrm{d} x=0, \\
& \lim _{j \rightarrow \infty} \int_{\Omega} q a(x)\left(\left|\frac{\nabla u_{h_{j}}}{\lambda_{h_{j}}}\right|^{q-2} \frac{\nabla u_{h_{j}}}{\lambda_{h_{j}}}-\left|\frac{\nabla u}{\lambda}\right|^{q-2} \frac{\nabla u}{\lambda}\right) \cdot \nabla\left(\frac{u_{h_{j}}}{\lambda_{h_{j}}}-\frac{u}{\lambda}\right) \mathrm{d} x=0,
\end{aligned}
$$

since, by convexity, the integrands are nonnegative. Now, we estimate the term

$$
\mathcal{R}_{q}:=\int_{\Omega} q a(x)\left(\left|\frac{\nabla u_{h_{j}}}{\lambda_{h_{j}}}\right|^{q-2} \frac{\nabla u_{h_{j}}}{\lambda_{h_{j}}}-\left|\frac{\nabla u}{\lambda}\right|^{q-2} \frac{\nabla u}{\lambda}\right) \cdot \nabla\left(\frac{u_{h_{j}}}{\lambda_{h_{j}}}-\frac{u}{\lambda}\right) \mathrm{d} x .
$$

We recall that the following inequalities hold for all $s, t \in \mathbb{R}^{n}$ (cf. inequalities (I) and (VII) of Section 10 of [39]),

$$
\begin{gathered}
|s-t|^{q} \leq 2^{q-2}\left(|s|^{q-2} s-|t|^{q-2} t\right) \cdot(s-t), \quad \text { if } q \geq 2, \\
|s-t|^{q} \leq(q-1)^{-\frac{q}{2}}\left[\left(|s|^{q-2} s-|t|^{q-2} t\right) \cdot(s-t)\right]^{\frac{q}{2}}\left(|s|^{2}+|t|^{2}\right)^{\frac{2-q}{2} \frac{q}{2}}, \quad \text { if } 1<q<2 .
\end{gathered}
$$

Therefore, if $q \geq 2$, by (3.32),

$$
\int_{\Omega} q a(x)\left|\nabla\left(\frac{u_{h_{j}}}{\lambda_{h_{j}}}-\frac{u}{\lambda}\right)\right|^{q} \mathrm{~d} x \leq 2^{q-2} \mathcal{R}_{q} .
$$

If $1<q<2$, Hölder's inequality gives

$$
\int_{\Omega}\left[q a(x)\left(\left|\frac{\nabla u_{h_{j}}}{\lambda_{h_{j}}}\right|^{q-2} \frac{\nabla u_{h_{j}}}{\lambda_{h_{j}}}-\left|\frac{\nabla u}{\lambda}\right|^{q-2} \frac{\nabla u}{\lambda}\right) \cdot \nabla\left(\frac{u_{h_{j}}}{\lambda_{h_{j}}}-\frac{u}{\lambda}\right)\right]^{\frac{q}{2}}
$$




$$
\begin{aligned}
& {\left[(q a(x))^{\frac{2}{q}}\left(\left|\frac{\nabla u_{h_{j}}}{\lambda_{h_{j}}}\right|^{2}+\left|\frac{\nabla u}{\lambda}\right|^{2}\right)\right]^{\frac{(2-q) q}{4}} \mathrm{~d} x } \\
\leq & \mathcal{R}_{q}^{\frac{q}{2}}\left[\int_{\Omega} q a(x)\left(\left|\frac{\nabla u_{h_{j}}}{\lambda_{h_{j}}}\right|^{2}+\left|\frac{\nabla u}{\lambda}\right|^{2}\right)^{\frac{q}{2}} \mathrm{~d} x\right]^{\frac{2-q}{2}} \\
\leq & \mathcal{R}_{q}^{\frac{q}{2}}\left[\int_{\Omega} q a(x)\left(\left|\frac{\nabla u_{h_{j}}}{\lambda_{h_{j}}}\right|^{q}+\left|\frac{\nabla u}{\lambda}\right|^{q}\right) \mathrm{d} x\right]^{\frac{2-q}{2}} \leq M \mathcal{R}_{q}^{\frac{q}{2}},
\end{aligned}
$$

where $M<\infty$ bounds from above the term in square brackets for all $j$, being $\left(\left|\nabla u_{h}\right| / \lambda_{h}\right)$ bounded in $L^{\mathcal{H}}(\Omega)$ and so in $L_{a}^{q}(\Omega)$. By (3.33), this implies that

$$
\int_{\Omega} q a(x)\left|\nabla\left(\frac{u_{h_{j}}}{\lambda_{h_{j}}}-\frac{u}{\lambda}\right)\right|^{q} \mathrm{~d} x \leq \frac{M}{(q-1)^{\frac{q}{2}}} \mathcal{R}_{q}^{\frac{q}{2}} .
$$

Analogous estimates as (3.34) and (3.35) hold for the term in $p$, therefore, by (3.31), this implies that

$$
\lim _{j \rightarrow \infty} \varrho_{\mathcal{H}}\left(\frac{\nabla u_{h_{j}}}{\lambda_{h_{j}}}-\frac{\nabla u}{\lambda}\right) \leq \lim _{j \rightarrow \infty} \int_{\Omega}\left(p\left|\frac{\nabla u_{h_{j}}}{\lambda_{h_{j}}}-\frac{\nabla u}{\lambda}\right|^{p}+q a(x)\left|\frac{\nabla u_{h_{j}}}{\lambda_{h_{j}}}-\frac{\nabla u}{\lambda}\right|^{q}\right) \mathrm{d} x=0 .
$$

By Lemma 2.1.11 of [23], the norm convergence and the modular convergence are equivalent in the space $L^{\mathcal{H}}(\Omega)$, so we obtain

$$
\frac{\nabla u_{h_{j}}}{\lambda_{h_{j}}} \rightarrow \frac{\nabla u}{\lambda}, \quad \text { in }\left[L^{\mathcal{H}}(\Omega)\right]^{n},
$$

and we can pass to the limit in the left-hand side of (3.26) under the integral sign (as it was already done for (3.29)) to obtain

$$
\begin{aligned}
& \lim _{j \rightarrow \infty} \int_{\Omega}\left[p\left(\frac{\left|\nabla u_{h_{j}}\right|}{\lambda_{h_{j}}}\right)^{p-2}+q a(x)\left(\frac{\left|\nabla u_{h_{j}}\right|}{\lambda_{h_{j}}}\right)^{q-2}\right] \frac{\nabla u_{h_{j}}}{\lambda_{h_{j}}} \cdot \nabla v \mathrm{~d} x \\
& \quad=\int_{\Omega}\left[p\left(\frac{|\nabla u|}{\lambda}\right)^{p-2}+q a(x)\left(\frac{|\nabla u|}{\lambda}\right)^{q-2}\right] \frac{\nabla u}{\lambda} \cdot \nabla v \mathrm{~d} x \text { for all } v \in C_{0}^{\infty}(\Omega) .
\end{aligned}
$$

Since $u_{h_{j}} \rightarrow u$ in $L^{\mathcal{H}}(\Omega)$ and $\frac{\nabla u_{h_{j}}}{\lambda_{h_{j}}} \rightarrow \frac{\nabla u}{\lambda}$ in $\left[L^{\mathcal{H}}(\Omega)\right]^{n}$, by dominated convergence we also get

$$
\lim _{j \rightarrow \infty} S\left(u_{h_{j}}\right)=\lim _{j \rightarrow \infty} \frac{\int_{\Omega}\left[p\left(\frac{\left|\nabla u_{h_{j}}\right|}{\lambda_{h_{j}}}\right)^{p}+q a(x)\left(\frac{\left|\nabla u_{h_{j}}\right|}{\lambda_{h_{j}}}\right)^{q}\right] \mathrm{d} x}{\int_{\Omega}\left[p\left|u_{h_{j}}\right|^{p}+q a(x)\left|u_{h_{j}}\right|^{q}\right] \mathrm{d} x}=S(u) .
$$

Together with (3.29) and (3.36), this proves claim, concluding the proof. 


\section{Variational eigenvalues}

Throughout this section, we assume that (1.7) holds and, unless explicitly stated, we consider $W_{0}^{1, \mathcal{H}}(\Omega)$ equipped with the $L^{\mathcal{H}}$-norm of the gradient.

Lemma 4.1 Let $u \in L^{\mathcal{H}}(\Omega) \backslash\{0\}$. For all $v \in L^{\mathcal{H}}(\Omega)$ the following inequality holds

$$
\left|\left\langle k^{\prime}(u), v\right\rangle\right| \leq q\|v\|_{\mathcal{H}} .
$$

Proof For $v=0$ the thesis is obvious, hence we suppose $v \neq 0$ and have by virtue of (3.5) and by Young's inequality

$$
\begin{aligned}
\left|\left\langle k^{\prime}(u), v\right\rangle\right| \leq & \|v\|_{\mathcal{H}} \int_{\Omega}\left[p\left(\frac{|u|}{k(u)}\right)^{p-1}+q a(x)\left(\frac{|u|}{k(u)}\right)^{q-1}\right] \frac{|v|}{k(v)} \mathrm{d} x \\
= & \|v\|_{\mathcal{H}}\left[\int_{\Omega} p^{(p-1) / p}\left(\frac{|u|}{k(u)}\right)^{p-1} p^{1 / p} \frac{|v|}{k(v)} \mathrm{d} x\right. \\
& \left.+\int_{\Omega}(q a(x))^{(q-1) / q}\left(\frac{|u|}{k(u)}\right)^{q-1}(q a(x))^{1 / q} \frac{|v|}{k(v)} \mathrm{d} x\right] \\
\leq & \|v\|_{\mathcal{H}}\left[\left(1-\frac{1}{p}\right) \int_{\Omega} p\left(\frac{|u|}{k(u)}\right)^{p} \mathrm{~d} x+\int_{\Omega}\left(\frac{|v|}{k(v)}\right)^{p} \mathrm{~d} x\right. \\
& \left.+\left(1-\frac{1}{q}\right) \int_{\Omega} q a(x)\left(\frac{|u|}{k(u)}\right)^{q} \mathrm{~d} x+\int_{\Omega} a(x)\left(\frac{|v|}{k(v)}\right)^{q} \mathrm{~d} x\right] \\
\leq & \|v\|_{\mathcal{H}}\left\{(q-1) \varrho_{\mathcal{H}}\left(\frac{u}{k(u)}\right)+\varrho_{\mathcal{H}}\left(\frac{v}{k(v)}\right)\right\}=q\|v\|_{\mathcal{H}} .
\end{aligned}
$$

This concludes the proof.

Theorem 4.2 $\widetilde{K}:=\left.K\right|_{\mathcal{M}}$ satisfies the $(P S)$ condition, i.e., every sequence $\left(u_{h}\right) \subset \mathcal{M}$ such that $\widetilde{K}\left(u_{h}\right) \rightarrow c$ for some $c \in \mathbb{R}$ and $\widetilde{K}^{\prime}\left(u_{h}\right) \rightarrow 0$ in $\left(W_{0}^{1, \mathcal{H}}(\Omega)\right)^{\prime}$ admits a convergent subsequence.

Proof By hypotheses there exist $c \in \mathbb{R}$ and a sequence $\left(c_{h}\right) \subset \mathbb{R}$ such that

$$
K\left(u_{h}\right) \rightarrow c \text { and } K^{\prime}\left(u_{h}\right)-c_{h} k^{\prime}\left(u_{h}\right) \rightarrow 0 \text { in }\left(W_{0}^{1, \mathcal{H}}(\Omega)\right)^{\prime} .
$$

It is easy to see that $\left\langle K^{\prime}\left(u_{h}\right), u_{h}\right\rangle=K\left(u_{h}\right)$ and $\left\langle k^{\prime}\left(u_{h}\right), u_{h}\right\rangle=k\left(u_{h}\right)=1$, so (4.1) implies that $c_{h} \rightarrow c$. Since $\left(u_{h}\right)$ is bounded in $W_{0}^{1, \mathcal{H}}(\Omega)$, up to a subsequence, $u_{h} \rightarrow u$ in $W_{0}^{1, \mathcal{H}}(\Omega)$ and $u_{h} \rightarrow u$ in $L^{\mathcal{H}}(\Omega)$. Thus, by Lemma 4.1 ,

$$
\left|\left\langle k^{\prime}\left(u_{h}\right), u_{h}-u\right\rangle\right| \leq q\left\|u_{h}-u\right\|_{\mathcal{H}} \rightarrow 0,
$$

and so the second limit in (4.1) implies

$$
\left\langle K^{\prime}\left(u_{h}\right), u_{h}-u\right\rangle \rightarrow 0
$$

as $h \rightarrow \infty$. Now, by the convexity of the $C^{1}$ functional $K$, we obtain for all $h$

$$
\left\|\nabla u_{h}\right\|_{\mathcal{H}} \leq\|\nabla u\|_{\mathcal{H}}+\left\langle K^{\prime}\left(u_{h}\right), u_{h}-u\right\rangle .
$$

Hence, (4.2) and the weak lower semicontinuity of the norm give

$$
\limsup _{h \rightarrow \infty}\left\|\nabla u_{h}\right\|_{\mathcal{H}} \leq\|\nabla u\|_{\mathcal{H}} \leq \liminf _{h \rightarrow \infty}\left\|\nabla u_{h}\right\|_{\mathcal{H}},
$$


whence

$$
\left\|\nabla u_{h}\right\|_{\mathcal{H}} \rightarrow\|\nabla u\|_{\mathcal{H}} \quad \text { as } h \rightarrow \infty .
$$

Clearly, also $\left\|u_{h}\right\|_{\mathcal{H}} \rightarrow\|u\|_{\mathcal{H}}$. Therefore, being $\left(W_{0}^{1, \mathcal{H}}(\Omega),\|\cdot\|_{1, \mathcal{H}}\right)$ uniformly convex by Proposition 2.14, we get $u_{h} \rightarrow u$ in $W_{0}^{1, \mathcal{H}}(\Omega)$ and conclude the proof.

The previous result allows us to define a sequence of eigenvalues of (3.3) by a minimax procedure.

Definition 4.3 For $m \in \mathbb{N}$, we define the $m$-th variational eigenvalue $\lambda_{\mathcal{H}}^{m}$ of (3.3) as

$$
\lambda_{\mathcal{H}}^{m}:=\inf _{K \in \mathcal{W}_{\mathcal{H}}^{m}} \sup _{u \in K}\|\nabla u\|_{\mathcal{H}}
$$

where $\mathcal{W}_{\mathcal{H}}^{m}$ is the set of compact subsets $K$ of $\mathcal{M}:=\left\{u \in W_{0}^{1, \mathcal{H}}(\Omega):\|u\|_{\mathcal{H}}=1\right\}$ that are symmetric (i.e., $K=-K$ ) and have topological index $i(K) \geq m$.

The topological index $i$ can be chosen as the Krasnosel'skil genus or the $\mathbb{Z}_{2}$-cohomological index of Fadell and Rabinowitz, and the results below hold with any index $i$ satisfying the following properties:

$\left(i_{1}\right)$ if $X$ is a topological vector space and $K \subseteq X \backslash\{0\}$ is compact, symmetric, and nonempty, $i(K)$ is an integer greater or equal than 1 ;

(i $\left.i_{2}\right)$ if $X$ is a topological vector space and $K \subseteq X \backslash\{0\}$ is compact, symmetric, and nonempty, then there exists an open subset $U$ of $X \backslash\{0\}$ such that $K \subseteq U$ and $i(\hat{K}) \leq i(K)$ for any compact, symmetric, and nonempty $\hat{K} \subseteq U$;

(i3) if $X, Y$ are two topological vector spaces, $K \subseteq X \backslash\{0\}$ is compact, symmetric, and nonempty and $\pi: K \rightarrow Y \backslash\{0\}$ is continuous and odd, we have $i(\pi(K)) \geq i(K)$;

$\left(i_{4}\right)$ if $(X,\|\cdot\|)$ is a normed space with $1 \leq \operatorname{dim} X<\infty$, then

$$
i(\{u \in X:\|u\|=1\})=\operatorname{dim} X .
$$

We remark that the new definition of $\lambda_{\mathcal{H}}^{1}$ is consistent with (3.2), by virtue of property $\left(i_{1}\right)$ and the fact that $K$ is an even functional.

- Proof of Theorem 1.2. By virtue of Theorem 4.2, we can apply Theorem 5.11 of [56] (see also Propositions 3.52 and 3.53 of [51]) to prove that the values defined in (4.3) are actually eigenvalues of (3.3) in the sense of Definition 3.3. Furthermore, since $\mathcal{W}_{\mathcal{H}}^{m+1} \subseteq \mathcal{W}_{\mathcal{H}}^{m}$ for all $m,\left(\lambda_{\mathcal{H}}^{m}\right)$ defines a nondecreasing sequence. Finally, $\lambda_{\mathcal{H}}^{m} \nearrow \infty$ as $m \rightarrow \infty$, being $i(\mathcal{M})=\infty$ by $\left(i_{4}\right)$ and $\left(i_{2}\right)$.

\section{Stability of variational eigenvalues}

In this section, we assume again the validity of condition $(1.7)$ and endow $W_{0}^{1, \mathcal{H}}(\Omega)$ with the $L^{\mathcal{H}}(\Omega)$-norm of the gradient.

For any couple of real numbers $(p, q)$ such that $1<p<q<n$, we define the corresponding $N$-function $\mathcal{H}(x, t):=t^{p}+a(x) t^{q}$ for all $(x, t) \in \Omega \times[0, \infty)$ and the related functionals $\mathscr{E}_{\mathcal{H}}: L^{1}(\Omega) \rightarrow[0, \infty]$ as

$$
\mathscr{E}_{\mathcal{H}}(u):= \begin{cases}\|\nabla u\|_{\mathcal{H}} & \text { if } u \in W_{0}^{1, \mathcal{H}}(\Omega), \\ +\infty & \text { otherwise }\end{cases}
$$


and $g_{\mathcal{H}}: L^{1}(\Omega) \rightarrow[0, \infty)$ as

$$
g_{\mathcal{H}}(u):= \begin{cases}\|u\|_{\mathcal{H}} & \text { if } u \in L^{\mathcal{H}}(\Omega), \\ 0 & \text { otherwise. }\end{cases}
$$

Proposition 5.1 The following properties hold:

(i) $g_{\mathcal{H}}$ is even and positively homogeneous of degree 1 ;

(ii) for every $b \in \mathbb{R}$ the restriction of $g_{\mathcal{H}}$ to $\left\{u \in L^{1}(\Omega): \mathscr{E}_{\mathcal{H}}(u) \leq b\right\}$ is continuous.

For the proof of Proposition 5.1, we refer the reader to Proposition 2.3 of [13], where a similar result is given in the case of functionals defined in variable exponent spaces.

Definition $5.2\left(\left(p_{h}, q_{h}\right)\right) \subset \mathbb{R}^{2}$ is an admissible nonincreasing sequence converging to $(p, q)$, and we shall write $\left(p_{h}, q_{h}\right) \searrow(p, q)$, if $q_{1}<n, p_{h}<q_{h}$ for all $h \in \mathbb{N}, p_{h} \searrow p$ and $q_{h} \searrow q$ as $h \rightarrow \infty$.

Lemma 5.3 Let $\left(\left(p_{h}, q_{h}\right)\right)$ be an admissible nonincreasing sequence converging to $(p, q)$. Then, for all $w \in C_{0}^{1}(\Omega)$,

$$
\lim _{h \rightarrow \infty}\|\nabla w\|_{\mathcal{H}_{h}}=\|\nabla w\|_{\mathcal{H}},
$$

where $\mathcal{H}_{h}$ is the $N$-function corresponding to the exponents $p_{h}$ and $q_{h}$ for all $h$.

Proof First, by Fatou's lemma we get that

$$
\begin{aligned}
\varrho_{\mathcal{H}}(\nabla w) & =\int_{\Omega}\left(|\nabla w|^{p}+a(x)|\nabla w|^{q}\right) \mathrm{d} x \leq \liminf _{h \rightarrow \infty} \int_{\Omega}\left(|\nabla w|^{p_{h}}+a(x)|\nabla w|^{q_{h}}\right) \mathrm{d} x \\
& =\liminf _{h \rightarrow \infty} \varrho_{\mathcal{H}_{h}}(\nabla w) .
\end{aligned}
$$

Now, let $\alpha:=\liminf _{h \rightarrow \infty}\|\nabla w\|_{\mathcal{H}_{h}}<\infty$ (in the case $\alpha=\infty$ this part of the proof is obvious) and take $\gamma>\alpha$. There exists a subsequence $\left(\left(p_{h_{j}}, q_{h_{j}}\right)\right)$ for which $\|\nabla w\|_{\mathcal{H}_{h_{j}}} \leq \gamma$ for all $j$, and so by the unit ball property and by (5.2)

$$
\varrho_{\mathcal{H}}\left(\frac{\nabla w}{\gamma}\right) \leq \liminf _{j \rightarrow \infty} \varrho_{\mathcal{H}_{h_{j}}}\left(\frac{\nabla w}{\gamma}\right) \leq 1
$$

and so $\|\nabla w\|_{\mathcal{H}} \leq \gamma$. By the arbitrariness of $\gamma$ we get

$$
\|\nabla w\|_{\mathcal{H}} \leq \liminf _{h \rightarrow \infty}\|\nabla w\|_{\mathcal{H}_{h}} .
$$

It remains to prove that

$$
\|\nabla w\|_{\mathcal{H}} \geq \limsup _{h \rightarrow \infty}\|\nabla w\|_{\mathcal{H}_{h}} .
$$

If $\|\nabla w\|_{\mathcal{H}}=0$ the conclusion follows immediately. Let us assume that $\|\nabla w\|_{\mathcal{H}}>0$ and take any $\gamma \in(0,1)$. By the boundedness of $\Omega$ we get

$$
\left|\frac{\gamma \nabla w}{\|\nabla w\|_{\mathcal{H}}}\right|^{p_{h}}+a(x)\left|\frac{\gamma \nabla w}{\|\nabla w\|_{\mathcal{H}}}\right|^{q_{h}} \leq(1+a(x))\left(1+\left|\frac{\gamma \nabla w}{\|\nabla w\|_{\mathcal{H}}}\right|^{n}\right) \in L^{1}(\Omega) .
$$

Hence, by the dominated convergence theorem and by (2.1.5) of [23]

$$
\begin{aligned}
\lim _{h \rightarrow \infty} \varrho_{\mathcal{H}_{h}}\left(\frac{\gamma \nabla w}{\|\nabla w\|_{\mathcal{H}}}\right) & =\int_{\Omega}\left(\left|\frac{\gamma \nabla w}{\|\nabla w\|_{\mathcal{H}}}\right|^{p}+a(x)\left|\frac{\gamma \nabla w}{\|\nabla w\|_{\mathcal{H}}}\right|^{q}\right) \mathrm{d} x=\varrho_{\mathcal{H}}\left(\frac{\gamma \nabla w}{\|\nabla w\|_{\mathcal{H}}}\right) \\
& \leq \gamma \varrho_{\mathcal{H}}\left(\frac{\nabla w}{\|\nabla w\|_{\mathcal{H}}}\right)=\gamma<1
\end{aligned}
$$


Therefore, for $h$ sufficiently large $\varrho_{\mathcal{H}_{h}}\left(\gamma \nabla w /\|\nabla w\|_{\mathcal{H}}\right)<1$ and by the unit ball property

$$
\left\|\frac{\gamma \nabla w}{\|\nabla w\|_{\mathcal{H}}}\right\|_{\mathcal{H}_{h}}<1
$$

that is $\|\nabla w\|_{\mathcal{H}_{h}}<\|\nabla w\|_{\mathcal{H}} / \gamma$. Whence,

$$
\limsup _{h \rightarrow \infty}\|\nabla w\|_{\mathcal{H}_{h}} \leq \frac{\|\nabla w\|_{\mathcal{H}}}{\gamma} \text { for all } \gamma \in(0,1),
$$

which implies (5.3).

Lemma 5.4 Put $\tilde{\mathcal{H}}(x, t):=t^{\tilde{p}}+a(x) t^{\tilde{q}}$ for all $(x, t) \in \Omega \times[0, \infty)$. If $p \leq \tilde{p}$ and $q \leq \tilde{q}$, with $1<\tilde{p}<\tilde{q}<n$, then $L^{\tilde{\mathcal{H}}}(\Omega) \hookrightarrow L^{\mathcal{H}}(\Omega)$, with constant embedding less than or equal to $|\Omega|+\|a\|_{1}+1$. If furthermore $\tilde{p}<2 p$ and $\tilde{q}<2 q$, the embedding constant is less than or equal to

$$
C_{\tilde{\mathcal{H}}, \mathcal{H}}:= \begin{cases}\frac{\tilde{p}-p}{p}\left(|\Omega|+\|a\|_{1}\right)+\left(\frac{\tilde{p}-p}{p}\right)^{\frac{p-\tilde{p}}{p}} & \text { if } \frac{\tilde{q}}{q} \leq \frac{\tilde{p}}{p}, \\ \frac{\tilde{q}-q}{q}\left(|\Omega|+\|a\|_{1}\right)+\left(\frac{\tilde{q}-q}{q}\right)^{\frac{q-\tilde{q}}{q}} & \text { otherwise. }\end{cases}
$$

In particular, if $\left(p_{h}, q_{h}\right) \searrow(p, q)$, then $C_{\mathcal{H}_{h}, \mathcal{H}} \rightarrow 1$ as $h \rightarrow \infty$, where $\mathcal{H}_{h}(x, t):=$ $t^{p_{h}}+a(x) t^{q_{h}}$.

Proof In what follows we shall use Young's inequality in this form,

$$
a b \leq \varepsilon a^{p^{\prime}}+C_{\varepsilon} b^{p} \quad \text { for all } a, b \geq 0, p>1, \varepsilon>0,
$$

with $p^{\prime}=p /(p-1)$ and $C_{\varepsilon}=\varepsilon^{-(p-1)}$. By (5.5), for all $(x, t) \in \Omega \times[0, \infty)$ and $\varepsilon>0$

$$
\begin{aligned}
& t^{p} \leq \varepsilon+\frac{1}{\varepsilon^{(\tilde{p}-p) / p}} t^{\tilde{p}} \\
& a(x) t^{q} \leq \varepsilon a(x)+\frac{a(x)}{\varepsilon^{(\tilde{q}-q) / q}} t^{\tilde{q}} .
\end{aligned}
$$

Thus,

$$
\mathcal{H}(x, t) \leq \varepsilon(1+a(x))+\frac{1}{\min \left\{\varepsilon^{(\tilde{p}-p) / p}, \varepsilon^{(\tilde{q}-q) / q}\right\}} \tilde{\mathcal{H}}(x, t)
$$

and in turn for all $u \in L^{\tilde{\mathcal{H}}}(\Omega)$

$$
\varrho_{\mathcal{H}}(u) \leq \varepsilon\left(|\Omega|+\|a\|_{1}\right)+\frac{1}{\min \left\{\varepsilon^{(\tilde{p}-p) / p}, \varepsilon^{(\tilde{q}-q) / q}\right\}} \varrho_{\tilde{\mathcal{H}}}(u) .
$$

First we put $\varepsilon:=1$ and we get for $u \neq 0$, by (5.6) and by the unit ball property,

$$
\varrho_{\mathcal{H}}\left(\frac{u}{\|u\|_{\tilde{\mathcal{H}}}}\right) \leq|\Omega|+\|a\|_{1}+1,
$$

and consequently, by (2.1.5) of [23],

$$
\varrho_{\mathcal{H}}\left(\frac{u}{\left(|\Omega|+\|a\|_{1}+1\right)\|u\|_{\tilde{\mathcal{H}}}}\right) \leq \frac{1}{|\Omega|+\|a\|_{1}+1} \varrho_{\mathcal{H}}\left(\frac{u}{\|u\|_{\tilde{\mathcal{H}}}}\right) \leq 1,
$$


which again by the unit ball property gives

$$
\|u\|_{\mathcal{H}} \leq\left(|\Omega|+\|a\|_{1}+1\right)\|u\|_{\tilde{\mathcal{H}}} \text { for all } u \in L^{\tilde{\mathcal{H}}}(\Omega) .
$$

For the second part of the statement, if $\tilde{q} / q \leq \tilde{p} / p$, we fix $\varepsilon:=(\tilde{p}-p) / p \in(0,1)$ and we get

$$
\min \left\{\varepsilon^{\frac{\tilde{p}-p}{p}}, \varepsilon^{\frac{\tilde{q}-q}{q}}\right\}=\left(\frac{\tilde{p}-p}{p}\right)^{\frac{\tilde{p}-p}{p}},
$$

otherwise we fix $\varepsilon:=\frac{\tilde{q}-q}{q} \in(0,1)$ and we get

$$
\min \left\{\varepsilon^{\frac{\tilde{p}-p}{p}}, \varepsilon^{\frac{\tilde{q}-q}{q}}\right\}=\left(\frac{\tilde{q}-q}{q}\right)^{\frac{\tilde{q}-q}{q}} .
$$

Now, by the unit ball property and by (5.6),

$$
\varrho_{\mathcal{H}}\left(\frac{u}{\|u\|_{\tilde{\mathcal{H}}}}\right) \leq C_{\tilde{\mathcal{H}}, \mathcal{H}} \quad \text { for all } u \in L^{\tilde{\mathcal{H}}}(\Omega) \backslash\{0\} .
$$

Therefore, being $\tilde{p}<2 p$ and $\tilde{q}<2 q, C_{\tilde{\mathcal{H}}, \mathcal{H}}>1$ and so

$$
\varrho_{\mathcal{H}}\left(\frac{u}{C_{\tilde{\mathcal{H}}, \mathcal{H}}\|u\|_{\tilde{\mathcal{H}}}}\right) \leq \frac{1}{C_{\tilde{\mathcal{H}}, \mathcal{H}}} \varrho_{\mathcal{H}}\left(\frac{u}{\|u\|_{\tilde{\mathcal{H}}}}\right) \leq 1 .
$$

The unit ball property then gives,

$$
\|u\|_{\mathcal{H}} \leq C_{\tilde{\mathcal{H}}, \mathcal{H}}\|u\|_{\tilde{\mathcal{H}}} \text { for all } u \in L^{\tilde{\mathcal{H}}}(\Omega),
$$

that is the thesis.

We now recall from [20] the notion of $\Gamma$-convergence that will be useful in the sequel.

Definition 5.5 Let $X$ be a metrizable topological space and let $\left(f_{h}\right)$ be a sequence of functions from $X$ to $\overline{\mathbb{R}}$. The $\Gamma$-lower limit and the $\Gamma$-upper limit of the sequence $\left(f_{h}\right)$ are the functions from $X$ to $\overline{\mathbb{R}}$ defined by

$$
\begin{aligned}
& \left(\Gamma-\liminf _{h \rightarrow \infty} f_{h}\right)(u)=\sup _{U \in \mathcal{N}(u)}\left[\liminf _{h \rightarrow \infty}\left(\inf \left\{f_{h}(v): v \in U\right\}\right)\right], \\
& \left(\Gamma-\limsup _{h \rightarrow \infty} f_{h}\right)(u)=\sup _{U \in \mathcal{N}(u)}\left[\limsup _{h \rightarrow \infty}\left(\inf \left\{f_{h}(v): v \in U\right\}\right)\right],
\end{aligned}
$$

where $\mathcal{N}(u)$ denotes the family of all open neighborhoods of $u$ in $X$. If there exists a function $f: X \rightarrow \overline{\mathbb{R}}$ such that

$$
\Gamma-\liminf _{h \rightarrow \infty} f_{h}=\Gamma-\limsup _{h \rightarrow \infty} f_{h}=f,
$$

then we write $\Gamma-\lim _{h \rightarrow \infty} f_{h}=f$ and we say that $\left(f_{h}\right) \Gamma$-converges to its $\Gamma$-limit $f$.

Theorem 5.6 If $\left(p_{h}, q_{h}\right) \searrow(p, q)$, then

$$
\mathscr{E}_{\mathcal{H}}(u)=\left(\Gamma-\lim _{h \rightarrow \infty} \mathscr{E}_{\mathcal{H}_{h}}\right)(u) \text { for all } u \in L^{1}(\Omega) .
$$


Proof Let $u \in L^{1}(\Omega)$. First we prove that

$$
\mathscr{E}_{\mathcal{H}}(u) \geq\left(\Gamma-\limsup _{h \rightarrow \infty} \mathscr{E}_{\mathcal{H}_{h}}\right)(u) .
$$

If $\mathscr{E}_{\mathcal{H}}(u)=\infty,(5.7)$ is immediate. Then, we suppose $\mathscr{E}_{\mathcal{H}}(u)<\infty$ and take $b \in \mathbb{R}, b>$ $\mathscr{E}_{\mathcal{H}}(u)$. Let $\delta>0$ and $w \in C_{0}^{1}(\Omega)$ be such that $\|u-w\|_{1}<\delta$, with $\|\nabla w\|_{\mathcal{H}}<b$. By Lemma 5.3 we get $\|\nabla w\|_{\mathcal{H}_{h}} \rightarrow\|\nabla w\|_{\mathcal{H}}$ and so

$$
b>\mathscr{E}_{\mathcal{H}}(w)=\lim _{h \rightarrow \infty}\|\nabla w\|_{\mathcal{H}_{h}}=\lim _{h \rightarrow \infty} \mathscr{E}_{\mathcal{H}_{h}}(w)
$$

and in turn

$$
b>\limsup _{h \rightarrow \infty}\left(\inf \left\{\mathscr{E}_{\mathcal{H}_{h}}(v):\|v-u\|_{1}<\delta\right\}\right) .
$$

By the arbitrariness of $b$, we conclude the proof of (5.7). Now, we want to prove that

$$
\mathscr{E}_{\mathcal{H}}(u) \leq\left(\Gamma-\liminf _{h \rightarrow \infty} \mathscr{E}_{\mathcal{H}_{h}}\right)(u) .
$$

Suppose that $\left(\Gamma-\liminf _{h \rightarrow \infty} \mathscr{E}_{\mathcal{H}_{h}}\right)(u)<\infty$ (otherwise the conclusion is obvious) and take $b \in \mathbb{R}, b>\left(\Gamma-\liminf _{h \rightarrow \infty} \mathscr{E}_{\mathcal{H}_{h}}\right)(u)$. By Proposition 8.1-(b) of [20] there is a sequence $\left(u_{h}\right) \subset L^{1}(\Omega)$ such that $u_{h} \rightarrow u$ in $L^{1}(\Omega)$ and

$$
\left(\Gamma-\liminf _{h \rightarrow \infty} \mathscr{E}_{\mathcal{H}_{h}}\right)(u)=\liminf _{h \rightarrow \infty} \mathscr{E}_{\mathcal{H}_{h}}\left(u_{h}\right) .
$$

Therefore, there exists a subsequence $\left(\left(p_{h_{j}}, q_{h_{j}}\right)\right)$ such that $\mathscr{E}_{\mathcal{H}_{h_{j}}}\left(u_{h_{j}}\right)<b$ for all $j$. Let $\left(v_{j}\right) \subset C_{0}^{1}(\Omega)$ be such that

$$
\left\|v_{j}-u_{h_{j}}\right\|_{1}<\frac{1}{j}, \quad \mathscr{E}_{\mathcal{H}_{h_{j}}}\left(v_{j}\right)<b \text { for all } j \in \mathbb{N} .
$$

Then, $v_{j} \rightarrow u$ in $L^{1}(\Omega)$ and by Lemma 5.4, for $j$ sufficiently large,

$$
b>\left\|\nabla v_{j}\right\|_{\mathcal{H}_{h_{j}}} \geq \frac{\left\|\nabla v_{j}\right\|_{\mathcal{H}}}{C_{\mathcal{H}_{h_{j}}, \mathcal{H}}} .
$$

Furthermore,

$$
\left\|\nabla v_{j}\right\|_{\mathcal{H}}<b\left(|\Omega|+\|a\|_{1}+1\right),
$$

thus $\left(v_{j}\right)$ is bounded in the reflexive Banach space $W_{0}^{1, \mathcal{H}}(\Omega)$, and so there exists a subsequence $\left(v_{j_{m}}\right)$ such that $v_{j_{m}} \rightarrow u$ in $W_{0}^{1, \mathcal{H}}(\Omega)$. By (5.9), by the weak lower semicontinuity of the norm, and by the fact that $C_{\mathcal{H}_{h_{j m}}, \mathcal{H}} \rightarrow 1$,

$$
b \geq \liminf _{m \rightarrow \infty} \frac{\left\|\nabla v_{j_{m}}\right\|_{\mathcal{H}}}{C_{\mathcal{H}_{h_{j_{m}}}, \mathcal{H}}} \geq\|\nabla u\|_{\mathcal{H}}=\mathscr{E}_{\mathcal{H}}(u) .
$$

Thus, (5.8) follows by the arbitrariness of $b$.

Lemma 5.7 Let $\left(p_{h}, q_{h}\right) \searrow(p, q)$, and $\left(u_{h}\right) \subset W_{0}^{1, \mathcal{H}}(\Omega)$ be a sequence such that

$$
\sup _{h \in \mathbb{N}}\left\|\nabla u_{h}\right\|_{\mathcal{H}}<\infty .
$$


Then, there exist a subsequence $\left(u_{h_{j}}\right)$ and a function $u \in W_{0}^{1, \mathcal{H}}(\Omega)$ such that

$$
\lim _{j \rightarrow \infty} \varrho \mathcal{H}_{h_{j}}\left(u_{h_{j}}\right)=\varrho_{\mathcal{H}}(u) .
$$

Proof By hypotheses $\left(u_{h}\right)$ is bounded in the reflexive Banach space $W_{0}^{1, \mathcal{H}}(\Omega)$, thus there exists a subsequence $\left(u_{h_{j}}\right)$ weakly convergent to $u \in W_{0}^{1, \mathcal{H}}(\Omega)$. By Proposition 2.15-(iii), $W_{0}^{1, \mathcal{H}}(\Omega) \hookrightarrow \hookrightarrow L^{p^{*}-\varepsilon}(\Omega)$ for $\varepsilon>0$ sufficiently small. Hence, $u_{h_{j}} \rightarrow u$ in $L^{p^{*}-\varepsilon}(\Omega)$ and, up to a subsequence,

$$
\begin{aligned}
& u_{h_{j}} \rightarrow u \text { a.e. in } \Omega, \\
& \left|u_{h_{j}}\right| \leq v \text { for all } j \in \mathbb{N}, \text { a.e. in } \Omega
\end{aligned}
$$

for some $v \in L^{p^{*}-\varepsilon}(\Omega)$. Now, for $j$ sufficiently large, if we take $\varepsilon<\left(p^{*}-q\right) / 2$, we get

$$
p_{h_{j}}<q_{h_{j}} \leq q+\varepsilon<p^{*}-\varepsilon
$$

and consequently

$$
\left|u_{h_{j}}\right|^{p_{h_{j}}}+a(x)\left|u_{h_{j}}\right|^{q_{h_{j}}} \leq 1+|v|^{p^{*}-\varepsilon}+\|a\|_{\infty}\left(1+|v|^{p^{*}-\varepsilon}\right) \in L^{1}(\Omega) .
$$

Finally, by the dominated convergence theorem, we obtain

$$
\lim _{j \rightarrow \infty} \int_{\Omega}\left(\left|u_{h_{j}}\right|^{p_{h_{j}}}+a(x)\left|u_{h_{j}}\right|^{q_{h_{j}}}\right) \mathrm{d} x=\int_{\Omega}\left(|u|^{p}+a(x)|u|^{q}\right) \mathrm{d} x .
$$

Theorem 5.8 Let $\left(p_{h}, q_{h}\right) \searrow(p, q)$. Then, for every subsequence $\left(\left(p_{h_{j}}, q_{h_{j}}\right)\right)$ and for every sequence $\left(u_{j}\right) \subset L^{1}(\Omega)$ verifying

$$
\sup _{j \in \mathbb{N}} \mathscr{E}_{\mathcal{H}_{j}}\left(u_{j}\right)<\infty
$$

there exists a subsequence $\left(u_{j_{m}}\right)$ such that, as $m \rightarrow \infty$

$$
\begin{aligned}
& u_{j_{m}} \rightarrow u \text { in } L^{1}(\Omega), \\
& g_{\mathcal{H}_{h_{j m}}}\left(u_{j_{m}}\right) \rightarrow g_{\mathcal{H}}(u) .
\end{aligned}
$$

Proof Put $b:=\sup _{j \in \mathbb{N}} \mathscr{E}_{\mathcal{H}_{h_{j}}}\left(u_{j}\right)$ and for all $j$ we get by Lemma 5.4

$$
\left\|\nabla u_{j}\right\|_{\mathcal{H}} \leq\left(|\Omega|+\|a\|_{1}+1\right) b<\infty,
$$

that is $\left(u_{j}\right)$ is bounded in the reflexive Banach space $W_{0}^{1, \mathcal{H}}(\Omega)$. Thus, there exists a subsequence $\left(u_{j_{m}}\right)$ such that $u_{j_{m}} \rightarrow u$ in $W_{0}^{1, \mathcal{H}}(\Omega), u_{j_{m}} \rightarrow u$ in $L^{1}(\Omega)$ by Lemma 2.15-(iii), and $u_{j_{m}} \rightarrow u$ a.e. in $\Omega$.

For the second part of the statement, we have to prove that $\left\|u_{j_{m}}\right\|_{\mathcal{H}_{h_{j m}}} \rightarrow\|u\|_{\mathcal{H}}$ up to a subsequence. In correspondence of

$$
\gamma>\liminf _{m}\left\|u_{j_{m}}\right\|_{\mathcal{H}_{h_{j m}}}
$$

we can find a subsequence, still denoted by $\left(\left(p_{h_{j_{m}}}, q_{h_{j_{m}}}\right)\right)$, for which $\left\|u_{j_{m}}\right\|_{\mathcal{H}_{h_{j m}}}<\gamma$ for all $m$. Therefore, $\varrho \mathcal{H}_{h_{j m}}\left(u_{j_{m}} / \gamma\right)<1$ and by Fatou's lemma

$$
\int_{\Omega}\left(\left|\frac{u}{\gamma}\right|^{p}+a(x)\left|\frac{u}{\gamma}\right|^{q}\right) \mathrm{d} x \leq \liminf _{m \rightarrow \infty} \int_{\Omega}\left(\left|\frac{u_{j_{m}}}{\gamma}\right|^{p_{h_{j m}}}+a(x)\left|\frac{u_{j_{m}}}{\gamma}\right|^{q_{h_{j m}}}\right) \mathrm{d} x \leq 1 .
$$


By the unit ball property $\|u\|_{\mathcal{H}} \leq \gamma$ and by the arbitrariness of $\gamma$, we conclude that

$$
\|u\|_{\mathcal{H}} \leq \liminf _{m}\left\|u_{j_{m}}\right\|_{\mathcal{H}_{h_{j m}}} .
$$

It remains to prove that $\|u\|_{\mathcal{H}} \geq \lim \sup _{m}\left\|u_{j_{m}}\right\|_{\mathcal{H}_{h_{j}}}$. Now, in correspondence of $\gamma<$ $\lim \sup _{m}\left\|u_{j_{m}}\right\|_{\mathcal{H}_{h_{j m}}}$, we can find a subsequence, still denoted by $\left(\left(p_{h_{j_{m}}}, q_{h_{j_{m}}}\right)\right)$, for which

$$
\left\|u_{j_{m}}\right\| \mathcal{H}_{h_{j m}}>\gamma
$$

and consequently $\varrho_{\mathcal{H}_{h_{j m}}}\left(u_{j_{m}} / \gamma\right)>1$ for all $m$. By Lemma 5.7, up to a subsequence

$$
\int_{\Omega}\left(\left|\frac{u}{\gamma}\right|^{p}+a(x)\left|\frac{u}{\gamma}\right|^{q}\right) \mathrm{d} x=\lim _{m \rightarrow \infty} \int_{\Omega}\left(\left|\frac{u_{j_{m}}}{\gamma}\right|^{p_{h_{j}}}+a(x)\left|\frac{u_{j_{m}}}{\gamma}\right|^{q_{h_{j}}}\right) \mathrm{d} x \geq 1 .
$$

We conclude the proof by using the unit ball property and the arbitrariness of $\gamma$ as before.

We are now ready to prove Theorem 1.3.

- Proof of Theorem 1.3. By Proposition 5.1 and by the definition of $\mathscr{E}_{\mathcal{H}}$, the functionals $\mathscr{E} \mathcal{H}$, $\mathscr{E}_{\mathcal{H}_{h}}, g_{\mathcal{H}}$ and $g_{\mathcal{H}_{h}}$ for all $h \in \mathbb{N}$ satisfy the structural assumptions required in Section 4 of [28]. Furthermore, Theorems 5.6 and 5.8 prove that all hypotheses of Corollary 4.4 of [28] are verified and consequently

$$
\lim _{h \rightarrow \infty} \inf _{K \in \mathcal{L}_{\mathcal{H}_{h}}^{m}} \sup _{u \in K} \mathscr{E}_{\mathcal{H}_{h}}=\inf _{K \in \mathcal{L}_{\mathcal{H}}^{m}} \sup _{u \in K} \mathscr{E}_{\mathcal{H}}
$$

where

$$
\mathcal{L}_{\mathcal{H}}^{m}:=\left\{K \subset L^{1}(\Omega) \cap\left\{\|u\|_{\mathcal{H}}=1\right\}: K \text { compact, } K=-K, i(K) \geq m\right\} \text { for all } m \in \mathbb{N}
$$

(here $i$ is defined with respect to the $L^{1}(\Omega)$-topology), and the sets $\mathcal{L}_{\mathcal{H}_{h}}^{m}$ are defined analogously for all $h \in \mathbb{N}$. Finally, by virtue of Proposition 5.1 (b), we can apply Corollary 3.3 of [28] to get that the minimax values with respect to the $L^{1}(\Omega)$-topology are the same as those with respect to the $W_{0}^{1, \mathcal{H}}(\Omega)$-topology and conclude the proof.

\section{A Weyl-type law}

Throughout this section, we assume that condition (1.7) is verified and that $\Omega$ is quasiconvex.

Lemma 6.1 For all $u \in W^{1, q}(\Omega) \backslash\{0\}$ we have

$$
\frac{1}{w} \cdot \frac{\|\nabla u\|_{p}}{\|u\|_{q}} \leq \frac{\|\nabla u\|_{\mathcal{H}}}{\|u\|_{\mathcal{H}}} \leq w \frac{\|\nabla u\|_{q}}{\|u\|_{p}}
$$

where $w:=1+\|a\|_{\infty}+|\Omega|$.

Proof For all $u \in L^{\mathcal{H}}(\Omega)$

$$
\int_{\Omega}|u|^{p} \mathrm{~d} x \leq \int_{\Omega}\left(|u|^{p}+a(x)|u|^{q}\right) \mathrm{d} x=\varrho_{\mathcal{H}}(u) .
$$

Hence, $\varrho_{\mathcal{H}}\left(u /\|u\|_{p}\right) \geq 1$ for $u \neq 0$, and by the unit ball property $\|u\|_{\mathcal{H}} \geq\|u\|_{p}$. On the other hand, for all $u \in L^{q}(\Omega)$

$$
\varrho_{\mathcal{H}}(u)=\int_{\Omega}\left(|u|^{p}+a(x)|u|^{q}\right) \mathrm{d} x \leq \int_{\Omega}\left[1+\left(1+\|a\|_{\infty}\right)|u|^{q}\right] \mathrm{d} x=|\Omega|+\left(1+\|a\|_{\infty}\right)\|u\|_{q}^{q}
$$


and so, if $u \neq 0$,

$$
\varrho_{\mathcal{H}}\left(\frac{u}{\|u\|_{q}}\right) \leq 1+\|a\|_{\infty}+|\Omega|,
$$

whence, by the unit ball property and being $1+\|a\|_{\infty}+|\Omega| \geq 1$,

$$
\|u\|_{\mathcal{H}} \leq\left(1+\|a\|_{\infty}+|\Omega|\right)\|u\|_{q} .
$$

Hence, for all $u \in L^{q}(\Omega)$

$$
\|u\|_{p} \leq\|u\|_{\mathcal{H}} \leq\left(1+\|a\|_{\infty}+|\Omega|\right)\|u\|_{q},
$$

which gives the thesis.

Lemma 6.2 Let $0<\delta<1$, consider the homothety $\Omega \rightarrow \delta \Omega, x \mapsto \delta x=: y$, and write $u(x)=v(y)$. Then, for all $u \in W^{1, q}(\Omega) \backslash\{0\}$

$$
\frac{\|\nabla v\|_{q}}{\|v\|_{p}}=\delta^{-\sigma-1} \frac{\|\nabla u\|_{q}}{\|u\|_{p}}, \quad \frac{\|\nabla v\|_{p}}{\|v\|_{q}}=\delta^{\sigma-1} \frac{\|\nabla u\|_{p}}{\|u\|_{q}},
$$

where $\sigma:=n\left(\frac{1}{p}-\frac{1}{q}\right)$.

The proof of the previous lemma follows by straightforward calculations. Furthermore, we notice that $\sigma \in(0,1)$, being $p<q<p^{*}$.

We introduce now the auxiliary problem in $W^{1, \mathcal{H}}(\Omega)$

$$
\begin{aligned}
-\operatorname{div}( & {\left.\left[p\left(\frac{|\nabla u|}{L(u)}\right)^{p-2}+q a(x)\left(\frac{|\nabla u|}{L(u)}\right)^{q-2}\right] \frac{\nabla u}{L(u)}\right) } \\
& =\lambda T(u)\left[p\left(\frac{|u|}{\ell(u)}\right)^{p-2}+q a(x)\left(\frac{|u|}{\ell(u)}\right)^{q-2}\right] \frac{u}{\ell(u)},
\end{aligned}
$$

where $L(u):=\|\nabla u\|_{\mathcal{H}}, \ell(u):=\|u\|_{\mathcal{H}}$, and

$$
T(u):=\frac{\int_{\Omega}\left[p\left(\frac{|\nabla u|}{L(u)}\right)^{p}+q a(x)\left(\frac{|\nabla u|}{L(u)}\right)^{q}\right] \mathrm{d} x}{\int_{\Omega}\left[p\left(\frac{|u|}{\ell(u)}\right)^{p}+q a(x)\left(\frac{|u|}{\ell(u)}\right)^{q}\right] \mathrm{d} x} .
$$

The eigenvalues and eigenfunctions of this problem on

$$
\mathcal{N}:=\left\{u \in W^{1, \mathcal{H}}(\Omega): \ell(u)=1\right\}
$$

are critical values and critical points of $\widetilde{L}:=\left.L\right|_{\mathcal{N}}$. Furthermore, we set for every $\lambda, \mu \in \mathbb{R}$

$$
\begin{aligned}
& \widetilde{K}^{\lambda}:=\{u \in \mathcal{M}: \widetilde{K}(u)<\lambda\}, \quad \widetilde{L}^{\mu}:=\{u \in \mathcal{N}: \widetilde{L}(u)<\mu\}, \\
& \widehat{K}(u):=\|\nabla u\|_{q} \text { for } u \in \widehat{\mathcal{M}}:=\left\{W_{0}^{1, q}(\Omega):\|u\|_{p}=1\right\}, \\
& \widehat{L}(u):=\|\nabla u\|_{p} \text { for } u \in \widehat{\mathcal{N}}:=\left\{u \in W^{1, q}(\Omega):\|u\|_{q}=1\right\}, \\
& \widehat{K}^{\lambda}:=\{u \in \widehat{\mathcal{M}}: \widehat{K}(u)<\lambda\} \text { and } \widehat{L}^{\mu}:=\{u \in \widehat{\mathcal{N}}: \widehat{L}(u)<\mu\} .
\end{aligned}
$$

For all $\lambda \in\left(\lambda_{\mathcal{H}}^{m}, \lambda_{\mathcal{H}}^{m+1}\right], i\left(\widetilde{K}^{\lambda}\right)=m$, (see Proposition 3.53 of [51] and [29]). Therefore,

$$
i\left(\widetilde{K}^{\lambda}\right)=\sharp\left\{m: \lambda_{\mathcal{H}}^{m}<\lambda\right\} \text { for all } \lambda \in \mathbb{R} .
$$


We recall here the definitions of two topological invariants of symmetric sets which will be useful in the next proofs.

Definition 6.3 For every nonempty and symmetric subset $A$ of a Banach space $X$, its cogenus is defined by

$$
\bar{\gamma}(A)=\inf \left\{k \in \mathbb{N}: \exists \text { a continuous odd map } f: \mathbb{S}^{k-1} \rightarrow A\right\},
$$

with the convention that $\bar{\gamma}(A):=+\infty$, if no such an integer $k$ exists.

Definition 6.4 ( $c f$. [25]) For every closed symmetric subset $A$ of a Banach space $X$, we define the quotient space $\bar{A}:=A / \mathbb{Z}_{2}$ with each $u$ and $-u$ identified. Let $F: \bar{A} \rightarrow \mathbb{R} P^{\infty}$ be the classifying map of $\bar{A}$ toward the infinite-dimensional projective space, which induces a homomorphism of the Alexander-Spanier cohomology rings $f^{*}: H^{*}\left(\mathbb{R} P^{\infty}\right) \rightarrow H^{*}(\bar{A})$. One can identify $H^{*}\left(\mathbb{R} P^{\infty}\right)$ with the polynomial ring $Z_{2}[\omega]$ on a single generator $\omega$. Finally, we define the cohomological index of $A$

$$
g(A):= \begin{cases}\sup \left\{k \in \mathbb{N}: f^{*}\left(\omega^{m-1}\right) \neq 0\right\}, & \text { if } A \neq \emptyset, \\ 0, & \text { if } A=\emptyset .\end{cases}
$$

In what follows the topological index $i$ used in definition (4.3) will be denoted by $\gamma$, when it stands for the Krasnosel'skil genus, and by $g$, when it stands for the $\mathbb{Z}_{2}$-cohomological index of Fadell and Rabinowitz.

By the monotonicity - property $\left(i_{2}\right)$ —and the supervariance-property $\left(i_{3}\right)$ —of the cohomological index, and by the fact that $g\left(\mathbb{S}^{k-1}\right)=k$, for any symmetric subset $A$ of a Banach space $X$, with finite genus, cogenus and cohomological index, it results

$$
\bar{\gamma}(A) \leq g(A) \leq \gamma(A) .
$$

Proposition $6.5 C^{\infty}(\bar{\Omega})$ is dense in $W^{1, \mathcal{H}}(\Omega)$.

Proof By Proposition 4.1 of [32], there exists a suitable extension $\tilde{\mathcal{H}}$ of $\mathcal{H}$ to all of $\mathbb{R}^{n} \times[0, \infty)$. For $u \in W^{1, \mathcal{H}}(\Omega)$, let $\tilde{u} \in W^{1, \tilde{\mathcal{H}}}\left(\mathbb{R}^{n}\right)$ denote an extension of $u$ such that

$$
\|\tilde{u}\|_{W^{1, \tilde{\mathcal{H}}}\left(\mathbb{R}^{n}\right)} \leq c\|u\|_{W^{1, \mathcal{H}}(\Omega)} .
$$

By Theorem 5.5 of [32], $C_{0}^{\infty}\left(\mathbb{R}^{n}\right)$ is dense in $W^{1, \tilde{\mathcal{H}}}\left(\mathbb{R}^{n}\right)$, so we can find $\left(\tilde{u}_{h}\right) \subset C_{0}^{\infty}\left(\mathbb{R}^{n}\right)$ such that $\tilde{u}_{h} \rightarrow \tilde{u}$ in $W^{1, \tilde{\mathcal{H}}}\left(\mathbb{R}^{n}\right)$. Therefore, put $u_{h}:=\left.\tilde{u}_{h}\right|_{\Omega}$ for all $h$ and have

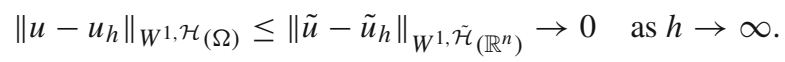

This proves that $u_{h}$ are the required approximating functions.

For an alternative proof of the previous result see also Theorem 2.6 of [6].

Lemma 6.6 For all $\lambda \in \mathbb{R}$

$$
\bar{\gamma}\left(\widehat{K}^{\lambda / w}\right) \leq \bar{\gamma}\left(\widetilde{K}^{\lambda}\right), \quad \gamma\left(\widetilde{L}^{\lambda}\right) \leq \gamma\left(\widehat{L}^{w \lambda}\right) .
$$

Proof By Lemma 6.1, the maps

$$
\widehat{K}^{\lambda / w} \rightarrow \widetilde{K}^{\lambda}, \quad u \mapsto \frac{u}{\|u\|_{\mathcal{H}}} \quad \text { and } \quad \widetilde{L}^{\lambda} \cap W^{1, q}(\Omega) \rightarrow \widehat{L}^{w \lambda}, \quad u \mapsto \frac{u}{\|u\|_{q}}
$$


are well defined, odd, and continuous. Moreover, by Proposition 6.5 and by the fact that $W^{1, q}(\Omega) \hookrightarrow W_{0}^{1, \mathcal{H}}(\Omega)$ (cf. Proposition 2.15- $(v)$ ), $W^{1, q}(\Omega)$ is dense in $W^{1, \mathcal{H}}(\Omega)$ and so the inclusion $\widetilde{L}^{\lambda} \cap W^{1, q}(\Omega) \subset \widetilde{L}^{\lambda}$ is a homotopy equivalence by virtue of Theorem 17 of [49]. The conclusion follows by the definition of $\gamma$ and $\bar{\gamma}$.

Lemma 6.7 If $\Omega_{1}$ and $\Omega_{2}$ are disjoint subdomains of $\Omega$ such that $\bar{\Omega}_{1} \cup \bar{\Omega}_{2}=\bar{\Omega}$, then

$$
\bar{\gamma}\left(\widehat{K}_{\Omega_{1}}^{\lambda}\right)+\bar{\gamma}\left(\widehat{K}_{\Omega_{2}}^{\lambda}\right) \leq \bar{\gamma}\left(\widehat{K}^{\lambda}\right), \quad \gamma\left(\widehat{L}^{\lambda}\right) \leq \gamma\left(\widehat{L}_{\Omega_{1}}^{\lambda^{\prime}}\right)+\gamma\left(\widehat{L}_{\Omega_{2}}^{\lambda^{\prime}}\right) \quad \text { for all } \lambda<\lambda^{\prime},
$$

where the subscripts indicate the corresponding domains and we drop the subscript when the domain is $\Omega$.

The proof of the previous lemma can be obtained reasoning as in the proof of Lemma 3.2 of [52], by simply replacing $p^{+}$with $q, p^{-}$with $p$ and $p(x)$ with $\mathcal{H}(x, t)$, hence we omit it.

We can now prove the last result of the paper. The proof relies on the argument produced for Theorem 1.1 of [52], we report it here for the sake of completeness.

- Proof of Theorem 1.4. The proof is split in two steps, first we prove the statement for $n$-dimensional cubes and then we approximate the domain $\Omega$ by unions of cubes.

Step 1. Let $Q$ be the unit cube in $\mathbb{R}^{n}$, fix $\lambda_{0}>\max \left\{\inf \widehat{K}_{Q}\right.$, inf $\left.\widehat{L}_{Q}\right\}$, and set

$$
r:=\bar{\gamma}\left(\widehat{K}_{Q}^{\lambda_{0}}\right), \quad s:=\gamma\left(\widehat{L}_{Q}^{\lambda_{0}}\right) .
$$

Then, take $\lambda \in\left(\lambda_{0}, \lambda^{\prime}\right)$ and any two cubes $Q_{a_{\lambda}}$ and $Q_{b_{\lambda^{\prime}}}$ of sides $a_{\lambda}:=\left(\lambda_{0} / \lambda\right)^{1 /(1+\sigma)}<1$ and $b_{\lambda^{\prime}}:=\left(\lambda_{0} / \lambda^{\prime}\right)^{1 /(1-\sigma)}<1$, respectively. By lemma 6.2 , it is easy to check that the functions

$$
\widehat{K}_{Q}^{\lambda_{0}} \rightarrow \widehat{K}_{Q_{a_{\lambda}}}^{\lambda}, \quad u \mapsto \frac{v}{\|v\|_{p}} \quad \text { and } \quad \widehat{L}_{Q}^{\lambda_{0}} \rightarrow \widehat{L}_{Q_{b_{\lambda^{\prime}}}^{\lambda^{\prime}}, \quad u \mapsto \frac{v}{\|v\|_{q}}}
$$

are odd homeomorphisms, and so, by property $\left(i_{3}\right)$ of the topological index, we obtain

$$
\bar{\gamma}\left(\widehat{K}_{Q_{a_{\lambda}}}^{\lambda}\right)=r, \quad \gamma\left(\widehat{L}_{Q_{b_{\lambda^{\prime}}}}^{\lambda^{\prime}}\right)=s .
$$

Therefore, by Lemma 6.7, if we denote by $Q_{a}$ a cube of side $a>0$,

$$
r\left[\frac{a}{a_{\lambda}}\right]^{n} \leq \bar{\gamma}\left(\widehat{K}_{Q_{a}}^{\lambda}\right), \quad \gamma\left(\widehat{L}_{Q_{a}}^{\lambda}\right) \leq s\left(\left[\frac{a}{b_{\lambda^{\prime}}}\right]+1\right)^{n},
$$

Whence, for $\lambda^{\prime}$ and $\lambda$ large

$$
C_{1} a^{n} \lambda^{n /(1+\sigma)} \leq \bar{\gamma}\left(\widehat{K}_{Q_{a}}^{\lambda}\right), \quad \gamma\left(\widehat{L}_{Q_{a}}^{\lambda}\right) \leq C_{2} a^{n}\left(\lambda^{\prime}\right)^{n /(1-\sigma)},
$$

with $C_{1}:=r / \lambda_{0}^{n /(1+\sigma)}$ and $C_{2}:=s / \lambda_{0}^{n /(1-\sigma)}$ depending only on $n, p$ and $q$.

Step 2. Let $\varepsilon>0$ and let $\Omega_{\varepsilon}, \Omega^{\varepsilon}$ be finite unions of cubes with pairwise disjoint interiors such that

$$
\Omega_{\varepsilon}:=\bigcup_{j=1}^{M_{\varepsilon}} Q_{j} \subset \Omega \subset \Omega^{\varepsilon}:=\bigcup_{j=1}^{M^{\varepsilon}} Q_{j}^{\prime}
$$

and $\left|\Omega^{\varepsilon} \backslash \Omega_{\varepsilon}\right|<\varepsilon$. Then, by (6.4), Lemma 6.7 and the monotonicity of $\bar{\gamma}$

$$
\begin{aligned}
& C_{1}\left|\Omega_{\varepsilon}\right| \lambda^{n /(1+\sigma)} \leq \sum_{j=1}^{M_{\varepsilon}} \bar{\gamma}\left(\widehat{K}_{Q_{j}}^{\lambda}\right) \leq \bar{\gamma}\left(\widehat{K}_{\Omega_{\varepsilon}}^{\lambda}\right) \leq \bar{\gamma}\left(\widehat{K}^{\lambda}\right), \\
& \gamma\left(\widehat{L}^{\lambda}\right) \leq \gamma\left(\widehat{L}_{\Omega^{\varepsilon}}^{\lambda}\right) \leq \sum_{j=1}^{M^{\varepsilon}} \gamma\left(\widehat{L}_{Q_{j}^{\prime}}^{\lambda^{\prime}}\right) \leq C_{2}\left|\Omega^{\varepsilon}\right|\left(\lambda^{\prime}\right)^{n /(1-\sigma)} .
\end{aligned}
$$


By Tietze theorem, we can extend continuously $a(\cdot)$ to all of $\mathbb{R}^{n}$ and obtain a nonnegative function having the same $L^{\infty}$-norm as $a(\cdot)$. Thus, by the arbitrariness of $\varepsilon>0$ and of $\lambda^{\prime}>\lambda$, we get

$$
\begin{aligned}
C_{1}|\Omega| \lambda^{n /(1+\sigma)} \leq \bar{\gamma}\left(\widehat{K}^{\lambda}\right) \leq \bar{\gamma}\left(\widetilde{K}^{w \lambda}\right) \leq g\left(\widetilde{K}^{w \lambda}\right) \\
\leq g\left(\widetilde{L}^{w \lambda}\right) \leq \gamma\left(\widetilde{L}^{w \lambda}\right) \leq \gamma\left(\widehat{L}^{w^{2} \lambda}\right) \leq C_{2}|\Omega|\left(w^{2} \lambda\right)^{n /(1-\sigma)},
\end{aligned}
$$

where we have used Lemma 6.6, inequalities (6.3), the fact that $\widetilde{K}^{\lambda} \subset \widetilde{L}^{\lambda}$, and the monotonicity of $g$. Finally, the conclusion follows by (6.1).

Acknowledgments The authors warmly thank Giuseppe Mingione for various suggestions about the problem and its related literature. Paolo Baroni is also acknowledged for some hints on the application of the Harnack inequality of [5] in the proof of Theorem 1.1. The statement and the proof of Lemma 2.12 are attributed to Lorenzo Brasco. Part of this work was written during a visit of Francesca Colasuonno at the Department of Computer Science of the University of Verona. The hosting institution is gratefully acknowledged. The research was partially supported by Gruppo Nazionale per l'Analisi Matematica, la Probabilità e le loro Applicazioni (INdAM).

\section{References}

1. Acerbi, E., Mingione, G.: Regularity results for a class of functionals with nonstandard growth. Arch. Ration. Mech. Anal. 156, 121-140 (2001)

2. Acerbi, E., Mingione, G.: Regularity results for a class of quasiconvex functionals with nonstandard growth. Ann. Scuola Norm. Sup. Pisa. 30, 311-339 (2001)

3. Baroni, P., Colombo, M., Mingione, G.: Non-autonomous Functionals, Borderline Cases and Related Function Classes. St. Petersburg Math. J. (2015, to appear)

4. Baroni, P., Kuusi, T., Mingione, G.: Borderline gradient continuity of minima. J. Fixed Point Theory Appl. 15, 537-575 (2014)

5. Baroni, P., Colombo, M., Mingione, G.: Harnack inequalities for double phase functionals. Nonlinear Anal. Theory Methods Appl. 121, 206-222 (2015)

6. Benkirane, A., Val, M.: Some approximation properties in Musielak-Orlicz-Sobolev spaces. Thai J. Math. 10, 371-381 (2012)

7. Bocea, M., Mihăilescu, M.: On the continuity of the Luxemburg norm of the gradient in $L^{p(\cdot)}$ with respect to $p(\cdot)$. Proc. Am. Math. Soc. 142, 507-517 (2014)

8. Brasco, L., Parini, E., Squassina, M.: Stability of variational eigenvalues for the fractional $p$-laplacian. Discrete Contin. Dyn. Syst. A 36, 1813-1845 (2016, to appear)

9. Brasco, L., Parini, E.: The second eigenvalue of the fractional p-laplacian, Adv. Calc. Var. (2015, to appear)

10. Brasco, L., Franzina, G.: An anisotropic eigenvalue problem of Stekloff type and weighted Wulff inequalities. Nonlinear Differ. Equ. Appl. 20, 1795-1830 (2013)

11. Brezis, H.: Functional Analysis, Sobolev Spaces and Partial Differential Equations. Springer, New York (2010)

12. Champion, T., De Pascale, L.: Asymptotic behaviour of nonlinear eigenvalue problems involving $p$ laplacian-type operators. Proc. R. Soc. Edinb. Sect. A 137, 1179-1195 (2007)

13. Colasuonno, F., Squassina, M.: Stability of eigenvalues for variable exponent problems. Nonlinear Anal. 124, 56-67 (2015)

14. Colombo, M., Mingione, G.: Bounded minimisers of double phase variational integrals. Arch. Ration. Mech. Anal. 218, 219-273 (2015)

15. Colombo, M., Mingione, G.: Regularity for double phase variational problems. Arch. Ration. Mech. Anal. 215, 443-496 (2015)

16. Courant, R., Hilbert, D.: Methods of Mathematical Physics, vol. I. New York (1953)

17. Cupini, G., Marcellini, P., Mascolo, E.: Regularity under sharp anisotropic general growth conditions. Discrete Contin. Dyn. Syst. B 11, 66-86 (2009)

18. Cupini, G., Marcellini, P., Mascolo, E.: Existence and regularity for elliptic equations under $p, q$-growth. Adv. Differ. Equ. 19, 693-724 (2014) 
19. Cupini, G., Marcellini, P., Mascolo, E.: Local boundedness of minimizers with limit growth conditions. J. Optim. Theory Appl. 166, 1-22 (2015)

20. Dal Maso, G.: An introduction to $\Gamma$-convergence. In: Progress in Nonlinear Differential Equations and their Applications, vol. 8. Birkhäuser, Boston (1993)

21. Degiovanni, M., Marzocchi, M.: Limit of minimax values under $\Gamma$-convergence. Electron. J. Differ. Equ. 2014, 1-19 (2014)

22. Degiovanni, M., Marzocchi, M.: On the dependence on $p$ of the variational eigenvalues of the $p$-Laplace operator. Potential Anal. Springer Netherlands, 1-17 (2015)

23. Diening, L., Harjulehto, P., Hästö, P., Růžička, M.: Lebesgue and Sobolev spaces with variable exponents, Lecture Notes in Mathematics, vol. 2017, Springer, Heidelberg (2011)

24. Faber, C.: Beweis dass unter allen homogen Membranen von gleicher Fläche und gleicher Spannung die kreisförmige den tiefsten Grundton gibt, Sitzungsber. Bayer. Akad. der Wiss. Math.-Phys., Munich, 169-172 (1923)

25. Fadell, E.R., Rabinowitz, P.H.: Generalized cohomological index theories for Lie group actions with an application to bifurcation questions for Hamiltonian systems. Invent. Math. 45, 139-174 (1978)

26. Fan, X.: An imbedding theorem for Musielak-Sobolev spaces. Nonlinear Anal. 75, 1959-1971 (2012)

27. Fan, X.: Differential equations of divergence form in Musielak-Sobolev spaces and sub-supersolution method. J. Math. Anal. Appl. 386, 593-604 (2012)

28. Franzina, G., Lindqvist, P.: An eigenvalue problem with variable exponents. Nonlinear Anal. 85, 1-16 (2013)

29. Friedlander, L.: Asymptotic behavior of the eigenvalues of the $\varphi$-Laplacian. Commun. Partial Differ. Equ. 14, 1059-1069 (1989)

30. Fusco, N., Maggi, F., Pratelli, A.: Stability estimates for certain Faber-Krahn, isocapacitary and Cheeger inequalities. Ann. Sc. Norm. Super. Pisa Cl. Sci. 8, 51-71 (2009)

31. García Azorero, J., Peral, I.: Comportement asymptotique des valeurs propres du $p$-laplacien. C. R. Acad. Sci. Paris Sér. I Math. 307, 75-78 (1988)

32. Harjulehto, P., Hästö, P., Klén, R.: Basic Properties of Generalized Orlicz Spaces, preprint

33. Iannizzotto, A., Squassina, M.: Weyl-type laws for fractional $p$-eigenvalue problems. Asymptot. Anal. 88, 233-245 (2014)

34. Juutinen, P., Lindqvist, P., Manfredi, J.J.: The $\infty$-eigenvalue problem. Arch. Ration. Mech. Anal. 148, 89-105 (1999)

35. Krahn, E.: Uber eine von Rayleigh formulierte Minimaleigenschaft des Kreises. Math. Ann. 94, 97-100 (1925)

36. Krahn, E.: Uber Minimaleigenschaft des Kugel in drei und mehr dimensionen. Acta Comment. Univ. Tartu Math. (Dorpat) A9, 1-44 (1926)

37. Lieb, E.: On the lowest eigenvalue of the Laplacian for the intersection of two domains. Invent. Math. 74, 441-448 (1983)

38. Lindgren, E., Lindqvist, P.: Fractional eigenvalues. Calc. Var. Partial Differ. Equ. 49, 795-826 (2014)

39. Lindqvist, P.: Notes on the p-Laplace equation, Report. University of Jyvaskyla Department of Mathematics and Statistics, 102 University of Jyvaskyla, Jyvaskyla (2006)

40. Lindqvist, P.: Stability for the solutions of $\operatorname{div}\left(|\nabla u|^{p-2} \nabla u\right)=f$ with varying $p$. J. Math. Anal. Appl. 127, 93-102 (1987)

41. Lindqvist, P.: On the equation $\operatorname{div}\left(|\nabla u|^{p-2} \nabla u\right)+\lambda|u|^{p-2} u=0$. Proc. Am. Math. Soc. 109, 157-164 (1990)

42. Lindqvist, P.: On non-linear Rayleigh quotients. Potential Anal. 2, 199-218 (1993)

43. Lindqvist, P.: Some remarkable sine and cosine functions. Ricerche Mat. 2, 269-290 (1995)

44. Lindqvist, P.: A nonlinear eigenvalue problem. Top. Math. Anal. 3, 175-203 (2008)

45. Marcellini, P.: Regularity of minimisers of integrals of the calculus of variations with non standard growth conditions. Arch. Ration. Mech. Anal. 105, 267-284 (1989)

46. Marcellini, P.: Regularity and existence of solutions of elliptic equations with $p, q$-growth conditions. J. Differ. Equ. 90, 1-30 (1991)

47. Marcellini, P.: Regularity for some scalar variational problems under general growth conditions. J. Optim. Theory Appl. 90, 161-181 (1996)

48. Musielak, J.: Orlicz spaces and modular spaces, Lecture Notes in Math. 1034, Springer, Berlin (1983)

49. Palais, R.S.: Homotopy theory of infinite dimensional manifolds. Topology 5, 1-16 (1966)

50. Parini, E.: Continuity of the variational eigenvalues of the $p$-Laplacian with respect to $p$. Bull. Aust. Math. Soc. 83, 376-381 (2011)

51. Perera, K., Agarwal, R.P., O’Regan, D.: Morse Theoretic Aspects of p-Laplacian Type Operators, vol. 161 of Mathematical Surveys and Monographs. American Mathematical Society, Providence (2010) 
52. Perera, K., Squassina, M.: Asymptotic behavior of the eigenvalues of the $p(x)$-Laplacian. Manuscripta Math. 144, 535-544 (2014)

53. Squassina, M.: On Ekeland's variational principle. J. Fixed Point Theory Appl. 10, 191-195 (2011)

54. Squassina, M.: Symmetry in variational principles and applications. J. Lond. Math. Soc. 85, 323-348 (2012)

55. Strutt, J.W.: (Lord Rayleigh), The Theory of Sound, MacMillan, New York, 1877. Dover, New York (1945)

56. Struwe, M.: Variational Methods, second edition: Ergebnisse Math. 34. Springer, Berlin (1996)

57. Van Schaftingen, J.: Symmetrization and minimax principles. Commun. Contemp. Math. 7, 463-481 (2005)

58. Weyl, H.: Das asymptotische Verteilungsgesetz der Eigenwerte linearer partieller Differentialgleichungen (mit einer Anwendung auf die Theorie der Hohlraumstrahlung). Math. Ann. 71, 441-479 (1912)

59. Yu, L.S.: Nonlinear $p$-Laplacian problems in unbounded domains. Proc. Am. Math. Soc. 115, 1037-1045 (1992)

60. Zhikov, V.V.: Averaging of functionals of the calculus of variations and elasticity theory. Izv. Akad. Nauk SSSR Ser. Mat. 50, 675-710 (1986)

61. Zhikov, V.V., Kozlov, S.M., Oleinik, O.A.: Homogenization of Differential Operators and Integral Functionals. Springer, Berlin (1994)

62. Zhikov, V.V.: On Lavrentiev's phenomenon. Russ. J. Math. Phys. 3, 249-269 (1995)

63. Zhikov, V.V.: On some variational problems. Russ. J. Math. Phys. 5, 105-116 (1997) 